\title{
ESTIMATION AND OPTIMAL PLANS OF MULTIPLE RAMP-STRESS ACCELERATED LIFE TESTS FOR THE GENERALIZED HALF-NORMAL DISTRIBUTION
}

\author{
A. M. Abd El-Raheem * \\ Department of Mathematics, Faculty of Education, \\ Ain Shams University, Cairo, Egypt
}

Received 18/3/2018 $\quad$ Revised 27/3/2018 Accepted 61/5/2018

\begin{abstract}
Accelerated life tests are commonly utilized in manufacturing industries to induce early failures of highly reliable products. In this paper, estimation and optimal design issues of multiple ramp-stress accelerated life tests are discussed for the generalized half-normal distribution. Assuming inverse power model as a life-stress relationship, the maximum likelihood estimates of the parameters, as well as Fisher information matrix are derived. In addition, the methods of least square, moments, Bayes are used for estimating the model parameters. The optimal proportion of test units allocated to each stress level is obtained under D and A-optimality criteria. A sensitivity analysis of the optimal allocation to misspecification of the model parameters is carried out. Furthermore, a real data set is used to show the application of the generalized half-normal distribution in reliability studies. Finally, a Monte Carlo simulation study is carried out to examine the performance of the estimation methods and the optimality criteria.
\end{abstract}

Keywords: accelerated life testing; optimal design; Fisher information matrix; generalized half-normal distribution; least square method; Bayes estimation; simulation study.

2000 AMS Classification:: 62N05, 62N02, 62K05.

\section{Introduction}

Due to the rapid development of the modern technology, the products and devices today become more reliable, and the products' life gets longer. For such highly reliable products, it is quite hard or even impossible to obtain the failure information under usual conditions. Therefore, to reduce the time required to obtain failure information, products should be tested at higher than usual levels of stress such test setting is called accelerated life testing (ALT). The failure time data from such ALTs are interpreted and analyzed to estimate the life characteristics under usual conditions. Three common methods of ALTs are constant-stress, step-stress and progressive-stress. The main difference between the three methods of ALTs is the relation between the stress loading and testing time. The stress is constant over the time in the constant-stress ALTs, but in the step-stress ALTs, the stress is increased step by step at prespecified times. While, in the progressive-stress ALTs, the stress is continuously increasing in time. If an ALT includes linearly increasing stress over the time, this test referred to as a ramp-stress test. ALTs can be categorized on the basic of the number of stress levels into two types: simple and multiple ALTs. Simple ALT contains only two stress levels while multiple ALT includes more than two stress levels. Some of the earlier works on ALTs include McCool 1, Miller and Nelson [2] and Nelson [3. Constant-stress, step-stress and progressive-stress models were studied by several authors; see Abdel-Hamid [4, Jaheen et al. 5] and Mohie El-Din et al. 6.7. for constant-stress ALTs. For step-stress ALTs; see Balakrishnan and Han 8 and Mohie El-Din et al. 9 11. For progressive-stress ALTs; see Abdel-Hamid and AL-Hussaini [12 13], AL-Hussaini et al. 14], Abdel-Hamid and Abushul [15]

* corresponding author: E-mail:a_m2am@yahoo.com 
and Mohie El-Din et al. 16.

The problems of planning optimal ALTs and making inferences were investigated by numerous authors. Miller and Nelson [2] initiated the investigation in this area by studying the optimal design of simple step-stress ALT for exponential distribution under uncensored data. Bai et al. [17] discussed the results of Miller and Nelson 22] in the case of censored data. Khamis and Higgins [18 studied the optimal plan of three stress levels for the exponential distribution. A comparison between constant and step-stress optimal design for Weibull failure data was done by Khamis [19]. Khamis and Higgins [20] introduced a new model for step-stress testing for Weibull distribution. Ng et al. 21] discussed the problem of specifying the optimal sample size allocation for extreme value distribution. Srivastava and Shukla [22] obtained the optimal plan for simple step-stress ALT under the log-logistic distribution by minimizing the asymptotic variance of the MLE of the median life at a design stress. Srivastava and Shukla 23 extended their results in 22$]$ to the case of censored data. Srivastava and Mittal 24] developed the results of Srivastava and Shukla 23 for truncated logistic distribution. Han and $\mathrm{Ng} 25$ introduced a comparative study between the optimal design of constant and step-stress ALT for exponential distribution under type-I censoring. Guan et al. 26] derived the optimal plans of constant-stress ALTs for the generalized exponential distribution. Han [27] considered time and cost constrained optimal designs of constant and step-stress ALTs for the exponential distribution. Mohie El-Din et al. 28 obtained the optimal designs of constant-stress ALTs for Lindley distribution. Abd El-Raheem 29 derived the optimal designs of constant-stress ALTs for the extension of the exponential distribution. Abd El-Raheem [30] expanded his results in Abd El-Raheem [29] to the censored data.

The generalized half-normal (GHN) distribution was derived from a reliable physical consideration of static fatigue model by Cooray and Ananda 31. This distribution displays more statistical attraction with an elastic thicker left tail than the existing lifetime distributions such as gamma, Weibull, lognormal, etc. Furthermore, the shapes of the hazard rate function such as increasing, decreasing, and bathtub make this distribution more applicable and a flexible lifetime distribution than the other known distributions. For different values of the parameters of GHN, positive skewness, as well as negative skewness being an excellent property of GHN distribution. This property makes GHN distribution the better fitness for the lifetime data. Many authors considered the GHN distribution as a life time model, see for example, Ahmadi et al. [32, Olmos et al. [33, and Wang and Shi [34. The probability density function (PDF) and cumulative distribution function (CDF) of $G H N(\theta, \alpha)$ distribution are given respectively as

$$
\begin{gathered}
f(t)=\sqrt{\frac{2}{\pi}}\left(\frac{\alpha}{t}\right)\left(\frac{t}{\theta}\right)^{\alpha} \exp \left\{-\frac{1}{2}\left(\frac{t}{\theta}\right)^{2 \alpha}\right\}, t>0, \alpha>0, \theta>0, \\
F(t)=2 \Phi\left[\left(\frac{t}{\theta}\right)^{\alpha}\right]-1, \quad t>0, \alpha>0, \theta>0
\end{gathered}
$$

where $\Phi($.$) is the CDF of the standard normal distribution.$

Notice that

$$
F(t)=2 \Phi\left[\left(\frac{t}{\theta}\right)^{\alpha}\right]-1=\Phi\left[\left(\frac{t}{\theta}\right)^{\alpha}\right]-\Phi\left[-\left(\frac{t}{\theta}\right)^{\alpha}\right]=1-2 \Phi\left[-\left(\frac{t}{\theta}\right)^{\alpha}\right] .
$$

Unfortunately, there are a few studies on the optimal design of ramp-stress ALTs as compared to constant and step-stress planning. Furthermore, the most recent studies on the optimal design of ramp-stress ALT considering the simple ramp-stress ALT; see for instance, Bai et al. 35, and Srivastava and Sharma 36. Thus, designing an effective multiple ramp-stress ALT for GHN distribution is required and of great attention. In this paper, the problems of estimation and optimal designs of multiple ramp-stress ALT for GHN distribution are studied. Under the assumption that the lifetime follows the GHN distribution and the scale parameter satisfies inverse power model, the proportion of test units allocated to each stress level are specified by D-criterion and A-criterion. Moreover, some inferring such as maximum likelihood estimates (MLEs), least square estimates (LSEs), moments estimates (MEs) and Bayes estimates (BEs) for the model parameters are investigated.

The article is planned as follows: In Section 2 test assumptions are exhibited. Some estimation techniques are discussed in Section 3 . The optimal proportion of test items assigned to each stress level is obtained under D and A-optimality criteria, in Section 4. In Section 5, a real data set is investigated to demonstrate the theoretical results in Sections 3 and 4 . In Section 6. the results obtained in Sections 3 and 4 are explained and compared using simulated data from the suggested model. The A-optimal design depends on the unknown model parameters, and for this cause, we study the sensitivity of the optimal design to misspecification of the parameters through Section 7. Lastly, conclusion is presented in Section 8 . 


\section{Test assumptions}

Assume $n$ be the overall number of specimens under the examination and $S_{1}(t)<\ldots<S_{k}(t)$ be the stress levels in the examination. Under every stress level $S_{i}(t), i=1,2, \ldots, k, n_{i}$ identical specimens are tested until all the $n_{i}$ units fail, such that $\sum_{i=1}^{k} n_{i}=n$. Suppose $t_{i 1}, t_{i 2}, \ldots, t_{i n_{i}}$ be the noticed failure times at stress level $S_{i}(t)$ such that $0<t_{i 1}<t_{i 2}<\ldots<$ $t_{i n_{i}}, i=1,2, \ldots, k$.

The goal here is to specify $n_{1}, n_{2}, \ldots, n_{k}$ according to some optimality criteria. The subsequent assumptions are used during the paper in the context of multiple ramp-stress ALT:

1. Under each stress level $S_{i}(t), i=1,2, \ldots, k, n_{i}=\Upsilon\left(n \pi_{i}\right)$ identical specimens are allocated under ramp-stress loading, where $\Upsilon($.$) is an approximate function, mapping its argument to a positive integer. To ensure \Upsilon\left(n \pi_{i}\right) \approx n \pi_{i}, \Upsilon($. could be one of round(.), floor(.), ceiling(.) and trunc(.). Since the above definition of $n_{i}$ complicates the distributional derivation of associated random quantities, for simplicity, we shall assume in all subsequent derivations that $n_{i} \equiv n \pi_{i}$, such that $\sum_{i=1}^{k} \pi_{i}=1, \sum_{i=1}^{k} n_{i}=n, 0 \leq \pi_{i} \leq 1$, where $\pi_{i}$ is the proportion of test units allocated to the stress level $S_{i}(t)$; see Han 27 .

2. Under every stress level $S_{i}(t)$, the lifetime of a unit follows $G H N\left(\theta_{i}, \alpha\right)$ distribution.

3. The progressive-stress $S_{i}(t)$ is directly proportional to the time with constant rate $\nu_{i}$, i.e. $S_{i}(t)=\nu_{i} t, \quad 0<\nu_{1}<\nu_{2}<$ $\ldots<\nu_{k}$.

4. The relationship between the life characteristic $\theta_{i}$ and the stress $S_{i}(t)$ holds the inverse power model, i.e.

$$
\theta_{i}(t)=\frac{1}{a\left[S_{i}(t)\right]^{b}}, i=1,2, \ldots, k,
$$

where $a, b$ are positive parameters should be estimated. For further information on this accelerated model; see Chapter 2 of Nelson [3].

5. The linear cumulative exposure model (LCEM) holds to demonstrate the impact of changing the stress from one level to another level, see Nelson [3, p. 507].

From the life-stress relationship in 2.1), the parameter $\theta_{i}$ can be expressed as

$$
\theta_{i}=\theta_{1} \psi_{i}^{b}, \quad i=1,2, \ldots, k,
$$

where $\theta_{1}$ is the scale parameter of the GHN distribution under the low-stress level $S_{1}(t)$ and $\psi_{i}=\frac{\nu_{1}}{\nu_{i}}, i=1,2, . ., k$ is the stress rate factor satisfying $0<\psi_{k}<\psi_{k-1}<\ldots<\psi_{2}<\psi_{1}=1$.

\subsection{Life distribution under multiple ramp-stress ALT}

From Equation (1.2) and using the LCEM, the CDF under multiple ramp-stress is given by, see Nelson [3, p. 507]

$$
G_{i}(t)=2 \Phi\left[\left(\frac{t}{\sigma_{i}}\right)^{\alpha}\right]-1, t>0
$$

the PDF of 2.3 is given by:

$$
g_{i}(t)=\sqrt{\frac{2}{\pi}}\left(\frac{\alpha}{t}\right)\left(\frac{t}{\sigma_{i}}\right)^{\alpha} \exp \left\{-\frac{1}{2}\left(\frac{t}{\sigma_{i}}\right)^{2 \alpha}\right\}, t>0,
$$

where $\sigma_{i}=(b+1) \theta_{1} \psi_{i}^{b}, i=1,2, \ldots, k$.

\section{Statistical inference}

In this section, some estimation techniques are applied to estimate the unknown parameters $\alpha, b$ and $\theta_{1}$ of PDF in (2.4). 


\subsection{Method of moments}

Let $t_{i j}$ be the observed values of the lifetime $T_{i}$ under progressive-stress level $S_{i}(t), i=1,2, \ldots, k$ and $j=1,2, \ldots, n_{i}$. The MEs $\left(\check{\alpha}, \breve{b}, \check{\theta_{1}}\right)$ of the parameters $\left(\alpha, b, \theta_{1}\right)$ can be obtained by solving the following three equations with respect to $\alpha, b$ and $\theta_{1}$.

$$
\sum_{i=1}^{k} E\left(T_{i}^{r}\right)=\sum_{i=1}^{k} \frac{1}{n_{i}} \sum_{j=1}^{n_{i}} t_{i j}^{r}, \quad r=1,2,3 .
$$

Based on the PDF in 2.4

$$
E\left(T_{i}^{r}\right)=\frac{\sqrt{2^{r / \alpha}} \Gamma\left(\frac{r+\alpha}{2 \alpha}\right)\left((b+1) \theta_{1} \psi_{i}^{b}\right)^{r}}{\sqrt{\pi}} .
$$

In spite of the simplicity of moment method, squaring and cubing of the sample observations can increase the sampling errors in the case of heavy-tailed situations. Outliers may also exist in the sample causing considerable distortion of the results.

\subsection{Least square estimation}

The least square technique is applied to estimate the unknown parameters by solving the normal equations of the sum of the squared deviations between the observed responses and the functional portion of the model. From (2.3) one has

$$
\log \left[\Phi^{-1}\left(\frac{1+G_{i}\left(t_{i j}\right)}{2}\right)\right]=\alpha \log \left(t_{i j}\right)-\beta_{0}-\beta_{1} \log \left(\psi_{i}\right),
$$

where $\beta_{0}=\alpha \log \left((b+1) \theta_{1}\right)$ and $\beta_{1}=\alpha b$.

Let $\hat{G}_{i}\left(t_{i j}\right)$ be the empirical distribution function of $G_{i}\left(t_{i j}\right)$ under $S_{i}(t)$, where $\hat{G}_{i}\left(t_{i j}\right)$ equals $j / n_{i}$. To avert $\log (0)$ in 3.2 , we change $\hat{G}_{i}\left(t_{i j}\right)$ to be $p_{i j}=j /\left(n_{i}+1\right)$. Therefore, the LSEs of $\beta_{0}, \beta_{1}$ and $\alpha$ can be obtained from

$$
Q\left(\beta_{0}, \beta_{1}, \alpha\right)=\sum_{i=1}^{k} \sum_{j=1}^{n_{i}}\left(x_{i j}-\alpha \log \left(t_{i j}\right)+\beta_{0}+\beta_{1} \log \left(\psi_{i}\right)\right)^{2},
$$

where $x_{i j}=\log \left[\Phi^{-1}\left(\left(1+p_{i j}\right) / 2\right)\right]$. Solving the normal equations

$$
\frac{\partial Q\left(\beta_{0}, \beta_{1}, \alpha\right)}{\partial \beta_{0}}=\frac{\partial Q\left(\beta_{0}, \beta_{1}, \alpha\right)}{\partial \beta_{1}}=\frac{\partial Q\left(\beta_{0}, \beta_{1}, \alpha\right)}{\partial \alpha}=0,
$$

we obtain

$$
\boldsymbol{A} \beta=\boldsymbol{B},
$$

where $\beta=\left(\beta_{0}, \beta_{1}, \alpha\right)^{\prime}$,

$$
\boldsymbol{A}=\left(\begin{array}{ccc}
\sum_{i=1}^{k} n_{i} & \sum_{i=1}^{k} n_{i} \psi_{i} & -\sum_{i=1}^{k} \sum_{j=1}^{n_{i}} \log \left(t_{i j}\right) \\
\sum_{i=1}^{k} n_{i} \psi_{i} & \sum_{i=1}^{k} n_{i} \psi_{i}^{2} & -\sum_{i=1}^{k} \sum_{j=1}^{n_{i}} \psi_{i} \log \left(t_{i j}\right) \\
\sum_{i=1}^{k} \sum_{j=1}^{n_{i}} \log \left(t_{i j}\right) & \sum_{i=1}^{k} \sum_{j=1}^{n_{i}} \psi_{i} \log \left(t_{i j}\right) & -\sum_{i=1}^{k} \sum_{j=1}^{n_{i}}\left(\log \left(t_{i j}\right)\right)^{2}
\end{array}\right),
$$

and

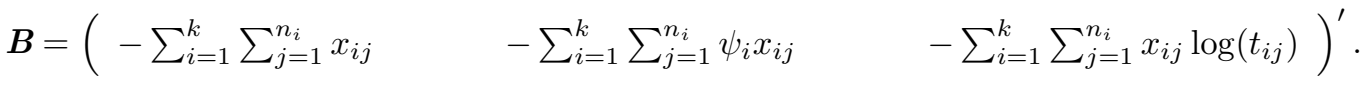

Hence the LSEs of $\beta$, say $\breve{\beta}=\left(\breve{\beta_{0}}, \breve{\beta_{1}}, \breve{\alpha}\right)^{\prime}$, can be obtained as $\breve{\beta}=\boldsymbol{A}^{-1} \boldsymbol{B}$, which indicates that the LSEs of $b$ and $\theta_{1}$ are given by $\breve{b}=\breve{\beta_{1}} / \breve{\alpha}$ and $\breve{\theta_{1}}=\frac{e^{\breve{\beta_{0}}} / \breve{\alpha}}{\frac{\hat{\beta}_{1}}{\check{\alpha}}+1}$.

As we mentioned for the moment method also, least square method is sensitivity to outliers. 


\subsection{Maximum likelihood estimation}

This portion considers the issue of obtaining the MLEs of the parameters $\alpha, b$ and $\theta_{1}$ and the associated Fisher information matrix for multiple ramp-stress ALT. From the PDF in 2.4), the likelihood function of the three parameters $\alpha, b$ and $\theta_{1}$ is obtained along these lines:

$$
L\left(\alpha, b, \theta_{1}\right)=\prod_{i=1}^{k} n_{i} ! \prod_{j=1}^{n_{i}} \sqrt{\frac{2}{\pi}}\left(\frac{\alpha}{t_{i j}}\right)\left(\frac{t_{i j}}{\sigma_{i}}\right)^{\alpha} \exp \left\{-\frac{1}{2}\left(\frac{t_{i j}}{\sigma_{i}}\right)^{2 \alpha}\right\},
$$

the log-likelihood function can be formulated as

$$
\ell\left(\alpha, b, \theta_{1}\right)=C+n \log (\alpha)-\alpha \sum_{i=1}^{k} n_{i} \log \left(\sigma_{i}\right)+(\alpha-1) \sum_{i=1}^{k} \sum_{j=1}^{n_{i}} \log \left(t_{i j}\right)-\frac{1}{2} \sum_{i=1}^{k} \sum_{j=1}^{n_{i}}\left(\frac{t_{i j}}{\sigma_{i}}\right)^{2 \alpha},
$$

where $C=\sum_{i=1}^{k} \log \left(n_{i} !\right)+\frac{n}{2} \log \left(\frac{2}{\pi}\right)$ and for $i=1,2, \ldots, k, \sigma_{i}=(b+1) \theta_{1} \psi_{i}^{b}$.

The likelihood equations of $\alpha, b$ and $\theta_{1}$ are respectively

$$
\begin{gathered}
\frac{\partial \ell}{\partial \alpha}=\frac{n}{\alpha}-\sum_{i=1}^{k} n_{i} \log \left(\sigma_{i}\right)+\sum_{i=1}^{k} \sum_{j=1}^{n_{i}} \log \left(t_{i j}\right)-\sum_{i=1}^{k} \sum_{j=1}^{n_{i}}\left(\frac{t_{i j}}{\sigma_{i}}\right)^{2 \alpha} \log \left(\frac{t_{i j}}{\sigma_{i}}\right) \\
\frac{\partial \ell}{\partial b}=-\frac{\alpha}{(b+1)} \sum_{i=1}^{k} n_{i}-\alpha \sum_{i=1}^{k} n_{i} \log \left(\psi_{i}\right)+\alpha \sum_{i=1}^{k} \sum_{j=1}^{n_{i}}\left(\frac{t_{i j}}{\sigma_{i}}\right)^{2 \alpha} \log \left(\psi_{i}\right)+\frac{\alpha}{(b+1)} \sum_{i=1}^{k} \sum_{j=1}^{n_{i}}\left(\frac{t_{i j}}{\sigma_{i}}\right)^{2 \alpha}, \\
\frac{\partial \ell}{\partial \theta_{1}}=-\frac{\alpha}{\theta_{1}} \sum_{i=1}^{k} n_{i}+\frac{\alpha}{\theta_{1}} \sum_{i=1}^{k} \sum_{j=1}^{n_{i}}\left(\frac{t_{i j}}{\sigma_{i}}\right)^{2 \alpha} .
\end{gathered}
$$

The MLEs $\hat{\alpha}, \hat{b}$ and $\hat{\theta_{1}}$ can be obtained by solving the system of nonlinear equations (3.6), 3.7) and (3.8).

The asymptotic normality distribution of MLEs can be used to obtain normal approximation confidence intervals (NACIs) of $\Theta=\left(\alpha, b, \theta_{1}\right)$. Miller 37 defined the asymptotic distribution of the MLEs of $\Theta$ as

$$
\left((\hat{\alpha}-\alpha),(\hat{b}-b),\left(\hat{\theta_{1}}-\theta_{1}\right)\right) \sim \mathbf{N}\left(0, \mathbf{F}^{-1}\left(\alpha, b, \theta_{1}\right)\right),
$$

where $\mathbf{F}^{-1}\left(\alpha, b, \theta_{1}\right)$ is the variance-covariance matrix of $\alpha, b$ and $\theta_{1}$.

$100(1-\rho) \%$ NACI of $\vartheta$ is given by

$$
\left(\hat{\vartheta}_{l}, \hat{\vartheta}_{u}\right)=\hat{\vartheta} \pm Z_{1-\rho / 2} \sqrt{\operatorname{Var}(\hat{\vartheta})}
$$

where $\vartheta$ is $\alpha, b$ or $\theta_{1}$, and $Z_{q}$ is the $100 q$ - th percentile of a standard normal distribution.

Theorem 3.1. According to the assumptions of the multiple ramp-stress ALT for GHN distribution, the Fisher information matrix of $\alpha, b$ and $\theta_{1}$ is

$$
\boldsymbol{F}\left(\alpha, b, \theta_{1}\right)=\left(\begin{array}{ccc}
\frac{n}{4 \alpha^{2}}\left[2(2-\log (2)-\gamma)^{2}+\pi^{2}-4\right] & \frac{-n}{\theta_{1}}(2-\log (2)-\gamma) & \frac{-(2-\log (2)-\gamma)}{(b+1)} \sum_{i=1}^{k} n_{i} \delta_{i} \\
\frac{-n}{\theta_{1}}(2-\log (2)-\gamma) & \frac{2 n \alpha^{2}}{\theta_{1}^{2}} & \frac{2 \alpha^{2}}{\theta_{1}(b+1)} \sum_{i=1}^{k} n_{i} \delta_{i} \\
\frac{-(2-\log (2)-\gamma)}{(b+1)} \sum_{i=1}^{k} n_{i} \delta_{i} & \frac{2 \alpha^{2}}{\theta_{1}(b+1)} \sum_{i=1}^{k} n_{i} \delta_{i} & \frac{2 \alpha^{2}}{(b+1)^{2}} \sum_{i=1}^{k} n_{i} \delta_{i}^{2}
\end{array}\right),
$$

where $\gamma$ is Euler's constant and $\delta_{i}=(b+1) \log \left(\psi_{i}\right)+1$. 
Proof. From (3.6), 3.7) and (3.8), we have

$$
\begin{gathered}
E\left(-\frac{\partial^{2} \ell}{\partial \alpha^{2}}\right)=\frac{n}{\alpha^{2}}+2 \sum_{i=1}^{k} n_{i} E\left(\left(\frac{T_{i}}{\sigma_{i}}\right)^{2 \alpha}\left(\log \left(\frac{T_{i}}{\sigma_{i}}\right)\right)^{2}\right), \\
E\left(-\frac{\partial^{2} \ell}{\partial b^{2}}\right)=-\frac{\alpha n}{(b+1)^{2}}+\frac{\alpha}{(b+1)^{2}} \sum_{i=1}^{k} n_{i} E\left(\left(\frac{T_{i}}{\sigma_{i}}\right)^{2 \alpha}\right)\left[2 \alpha\left((b+1) \log \psi_{i}+1\right)^{2}+1\right], \\
E\left(-\frac{\partial^{2} \ell}{\partial \theta_{1}^{2}}\right)=-\frac{\alpha n}{\theta_{1}^{2}}+\frac{\alpha(2 \alpha+1)}{\theta_{1}^{2}} \sum_{i=1}^{k} n_{i} E\left(\left(\frac{T_{i}}{\sigma_{i}}\right)^{2 \alpha}\right), \\
\left.-\frac{1}{\partial \alpha \partial b}\right)=\frac{n}{(b+1)}+\sum_{i=1}^{k} n_{i} \log \left(\psi_{i}\right)-\sum_{i=1}^{k} n_{i} E\left(\left(\frac{T_{i}}{\sigma_{i}}\right)^{2 \alpha}\right) \log \left(\psi_{i}\right)-2 \alpha \sum_{i=1}^{k} n_{i} E\left(\left(\frac{T_{i}}{\sigma_{i}}\right)^{2 \alpha}\right)-\frac{2 \alpha}{(b+1)} \sum_{i=1}^{k} n_{i} E\left(\left(\frac{T_{i}}{\sigma_{i}}\right)^{2 \alpha} \log \left(\frac{T_{i}}{\sigma_{i}}\right)\right) \\
E\left(-\frac{\partial^{2} \ell}{\partial \alpha \partial \theta_{1}}\right)=\frac{n}{\theta_{1}}-\frac{1}{\theta_{1}} \sum_{i=1}^{k} n_{i} E\left(\left(\frac{T_{i}}{\sigma_{i}}\right)^{2 \alpha}\right)-\frac{2 \alpha}{\theta_{1}} \sum_{i=1}^{k} n_{i} E\left(\left(\frac{T_{i}}{\sigma_{i}}\right)^{2 \alpha} \log \left(\frac{T_{i}}{\sigma_{i}}\right)\right) \\
E\left(-\frac{\partial^{2} \ell}{\partial b \partial \theta_{1}}\right)=\frac{2 \alpha^{2}}{\theta_{1}(b+1)} \sum_{i=1}^{k} n_{i} E\left(\left(\frac{T_{i}}{\sigma_{i}}\right)^{2 \alpha}\right)\left[1+(b+1) \log \left(\psi_{i}\right)\right] .
\end{gathered}
$$

From (2.4), one has

$$
\begin{gathered}
E\left(\left(\frac{T_{i}}{\sigma_{i}}\right)^{2 \alpha}\right)=1, \\
E\left(\left(\frac{T_{i}}{\sigma_{i}}\right)^{2 \alpha} \log \left(\frac{T_{i}}{\sigma_{i}}\right)\right)=\frac{1}{2 \alpha}(2-\log (2)-\gamma), \\
E\left(\left(\frac{T_{i}}{\sigma_{i}}\right)^{2 \alpha}\left(\log \left(\frac{T_{i}}{\sigma_{i}}\right)\right)^{2}\right)=\frac{1}{4 \alpha^{2}}\left[(2-\log (2)-\gamma)^{2}+\frac{\pi^{2}}{2}-4\right] .
\end{gathered}
$$

Then by some algebraic computations, we get the Fisher information matrix as in 3.10 .

\subsection{Bayes estimation}

BEs of the model parameters $\alpha, b$ and $\theta_{1}$ are calculated according to square error (SE) and linear exponential (LINEX) loss functions. Consider the model parameters $\alpha, b$ and $\theta_{1}$ are independent and have priors along these lines:

$$
\begin{gathered}
P_{1}(\alpha) \propto \alpha^{\mu_{1}-1} e^{-\lambda_{1} \alpha .}, \quad \alpha>0, \mu_{1}, \lambda_{1}>0, \\
P_{2}(b) \propto b^{\mu_{2}-1} e^{-\lambda_{2} b}, \quad b>0, \mu_{2}, \lambda_{2}>0, \\
P_{3}\left(\theta_{1}\right) \propto \theta_{1}^{\mu_{3}-1} e^{-\lambda_{3} \theta_{1}}, \quad \theta_{1}>0, \mu_{3}, \lambda_{3}>0 .
\end{gathered}
$$

The non-informative priors (NIPs) case can be obtained when $\mu_{i}=\lambda_{i} \rightarrow 0, i=1,2,3$. From (3.17), (3.18) and (3.19), the joint prior of the parameters $\alpha, b$ and $\theta_{1}$ is

$$
P\left(\alpha, b, \theta_{1}\right) \propto \alpha^{\mu_{1}-1} b^{\mu_{2}-1} \theta_{1}^{\mu_{3}-1} e^{-\left(\alpha \lambda_{1}+b \lambda_{2}+\theta_{1} \lambda_{3}\right)}, \quad \alpha, b, \theta_{1}>0 .
$$


From (3.4) and (3.20), the joint posterior density function of the parameters $\alpha, b$ and $\theta_{1}$ can be formulated as follows:

$$
P^{*}\left(\alpha, b, \theta_{1}\right) \propto \alpha^{\sum_{i=1}^{k} n_{i}+\mu_{1}-1} b^{\mu_{2}-1} \theta_{1}^{\mu_{3}-1} \exp \left\{-\alpha \lambda_{1}-b \lambda_{2}-\theta_{1} \lambda_{3}-\frac{1}{2} \sum_{i=1}^{k} \sum_{j=1}^{n_{i}}\left(\frac{t_{i j}}{\sigma_{i}}\right)^{2 \alpha}\right\} \prod_{i=1}^{k} \prod_{j=1}^{n_{i}} \frac{1}{\sigma_{i}}\left(\frac{t_{i j}}{\sigma_{i}}\right)^{\alpha-1} .
$$

The BEs of the function of parameters $U(\Theta)=U\left(\alpha, b, \theta_{1}\right)$ under SE and LINEX loss functions are respectively

$$
\widetilde{U}_{S E}(\Theta)=\int_{\Theta} U(\Theta) P^{*}(\Theta) d \Theta
$$

and

$$
\widetilde{U}_{L I N E X}(\Theta)=-\frac{1}{c} \log \left[\int_{\Theta} e^{-c U(\Theta)} P^{*}(\Theta) d \Theta\right],
$$

where $c \neq 0$ is the shape parameter of LINEX loss function.

Regrettably, we cannot calculate the integrations in $(3.22)$ and $(3.23)$ explicitly. As a result, Markov chain Monte Carlo (MCMC) technique is applied to approximate these integrations. From the joint posterior density function in (3.21), the conditional posterior distributions of $\alpha, b$ and $\theta_{1}$ are given respectively by

$$
\begin{gathered}
P^{*}\left(\alpha \mid b, \theta_{1}\right) \propto \alpha^{\sum_{i=1}^{k} n_{i}+\mu_{1}-1} \exp \left\{-\alpha \lambda_{1}-\frac{1}{2} \sum_{i=1}^{k} \sum_{j=1}^{n_{i}}\left(\frac{t_{i j}}{\sigma_{i}}\right)^{2 \alpha}\right\} \prod_{i=1}^{k} \prod_{j=1}^{n_{i}}\left(\frac{t_{i j}}{\sigma_{i}}\right)^{\alpha}, \\
P^{*}\left(b \mid \alpha, \theta_{1}\right) \propto b^{\mu_{2}-1} \exp \left\{-b \lambda_{2}-\frac{1}{2} \sum_{i=1}^{k} \sum_{j=1}^{n_{i}}\left(\frac{t_{i j}}{\sigma_{i}}\right)^{2 \alpha}\right\} \prod_{i=1}^{k} \prod_{j=1}^{n_{i}}\left((b+1) \psi_{i}^{b}\right)^{-\alpha} \\
P^{*}\left(\theta_{1} \mid \alpha, b\right) \propto \theta_{1}^{-\alpha \sum_{i=1}^{k} n_{i}+\mu_{3}-1} \exp \left\{-\theta_{1} \lambda_{3}-\frac{1}{2} \sum_{i=1}^{k} \sum_{j=1}^{n_{i}}\left(\frac{t_{i j}}{\sigma_{i}}\right)^{2 \alpha}\right\} .
\end{gathered}
$$

We cannot simplify (3.24), (3.25) and (3.26) to popular distribution. Subsequently, Metropolis algorithm with normal proposal distribution $\mathbf{N}(.$, .) is utilized to simulate samples from these distribution. The following algorithm is suggested to evaluate BEs of $U=U\left(\alpha, b, \theta_{1}\right)$ under SE and LINEX loss functions.

\section{Algorithm(1)}

1. Begin with initial guess point of $\left(\alpha, b, \theta_{1}\right)$ say $\left(\alpha^{(0)}, b^{(0)}, \theta_{1}^{(0)}\right)$.

2. Set $i=1$.

3. Generate $\alpha^{*}$ from proposal distribution $\mathbf{N}\left(\alpha^{(i-1)}, \operatorname{var}\left(\alpha^{(i-1)}\right)\right)$.

4. Obtain the acceptance probability

$$
r\left(\alpha^{(i-1)} \mid \alpha^{*}\right)=\min \left[1, \frac{P^{*}\left(\alpha^{*} \mid b^{(i-1)}, \theta_{1}^{(i-1)}\right)}{P^{*}\left(\alpha^{(i-1)} \mid b^{(i-1)}, \theta_{1}^{(i-1)}\right)}\right] .
$$

5. Generate $U \sim U(0,1)$.

6. If $U \leq r\left(\alpha^{(i-1)} \mid \alpha^{*}\right)$, approve the proposal distribution and set $\alpha^{(i)}=\alpha^{*}$. Otherwise, refuse the proposal distribution and set $\alpha^{(i)}=\alpha^{(i-1)}$.

7. To generate $b^{*}$, perform the steps ((2)-(6)) for $b$.

8. To generate $\theta_{1}^{*}$, perform the steps $((2)-(6))$ for $\theta_{1}$.

9. Set $i=i+1$. 
10. Duplicate steps ((3)-(9)), $N$ times.

11. Evaluate the BEs of $\alpha, b$ and $\theta_{1}$ using MCMC under SE loss function as

$$
\tilde{\vartheta}=\frac{1}{N-M} \sum_{i=M+1}^{N} \vartheta^{(i)}, \quad \text { where } \vartheta \text { is } \alpha, b \text { or } \theta_{1},
$$

where $M$ is the burn-in period.

12. Evaluate the BEs of $\alpha, b$ and $\theta_{1}$ using MCMC under LINEX loss function as

$$
\tilde{\vartheta}=-\frac{1}{c} \log \left[\frac{1}{N-M} \sum_{i=M+1}^{N} e^{-c \vartheta^{(i)}}\right], \quad \text { where } \vartheta \text { is } \alpha, b \text { or } \theta_{1} .
$$

13. Sort the posterior sample $\left\{\vartheta^{(i)}, i=M+1, \ldots, N\right\}$ to obtain the ordered values as $\left\{\vartheta^{[1]}, \vartheta^{[2]}, \ldots, \vartheta^{[N-M]}\right\}$. Then, the $100(1-\rho) \%$ Bayesian credible interval (BCI) of $\vartheta$ is given by

$$
\left(\tilde{\vartheta}_{l}, \tilde{\vartheta}_{u}\right)=\left(\vartheta^{[\rho(N-M) / 2]}, \vartheta^{[(1-\rho / 2)(N-M)]}\right), \quad \text { where } \vartheta \text { is } \alpha, b \text { or } \theta_{1} .
$$

\section{Optimal ramp-stress ALT plans}

The optimal proportion $\Pi^{*}=\left(\pi_{1}^{*}, \pi_{2}^{*}, \ldots, \pi_{k}^{*}\right)$ of test units allocated to each stress level is obtained under $\mathrm{D}$ and Aoptimality criteria.

\subsection{D-optimality}

The D-optimality criterion is frequently used in designing ALT by maximizing the determinant of the Fisher information matrix. Therefore, our aim is to obtain the optimal proportions $\pi_{1}^{*}, \pi_{2}^{*}, \ldots, \pi_{k}^{*}$ to maximize the determinant of the Fisher information matrix $\left|\mathbf{F}\left(\alpha, b, \theta_{1}\right)\right|$.

Based on 3.10 , one has

$$
\left|\mathbf{F}\left(\alpha, b, \theta_{1}\right)\right|=\frac{n^{3} \alpha^{2}\left(\pi^{2}-4\right)}{(b+1)^{2} \theta_{1}^{2}}\left[\sum_{i=1}^{k} \pi_{i} \delta_{i}^{2}-\left(\sum_{i=1}^{k} \pi_{i} \delta_{i}\right)^{2}\right] .
$$

For a multiple ramp-stress ALT, maximizing $\left|\mathbf{F}\left(\alpha, b, \theta_{1}\right)\right|$ is equivalent to selecting $\pi_{1}, \pi_{2}, \ldots, \pi_{k}$ for maximizing the objective function

$$
\phi(\Pi)=\left[\sum_{i=1}^{k} \pi_{i} \delta_{i}^{2}-\left(\sum_{i=1}^{k} \pi_{i} \delta_{i}\right)^{2}\right] .
$$

It is very interesting that 4.2 is independent on the parameters $\alpha, b$ and $\theta_{1}$. In other words, the optimal proportions $\pi_{1}^{*}, \pi_{2}^{*}, \ldots, \pi_{k}^{*}$ are same for any values of $\alpha, b$ and $\theta_{1}$. That is, the optimal plan is very robust.

Theorem 4.1. The D-optimality allocation proportions of a simple ramp-stress ALT for GHN distribution under complete sampling are $\pi_{1}^{*}=\frac{1}{2}$ and $\pi_{2}^{*}=\frac{1}{2}$.

Proof. In the case of a simple ramp-stress ALT, $k=2$ and $\pi_{2}=1-\pi_{1}$, the objective function in 4.2 becomes

$$
\phi\left(\pi_{1}\right)=\pi_{1}+\left(1-\pi_{1}\right) \delta_{2}^{2}-\left(\pi_{1}+\left(1-\pi_{1}\right) \delta_{2}\right)^{2},
$$

to maximize $\phi\left(\pi_{1}\right)$ with respect to $\pi_{1}$, solving $\frac{d \phi\left(\pi_{1}\right)}{d \pi_{1}}=0$, gives $\pi_{1}^{*}=\frac{1}{2}$ as a root of this equation and consequently $\pi_{2}^{*}=\frac{1}{2}$.

Theorem 4.2. The D-optimality allocation proportions of a multiple ramp-stress ALT for GHN distribution under complete sampling are $\pi_{1}^{*}=\frac{1}{2}, \pi_{i}^{*}=0, \forall i=2,3, \ldots, k-1$ and $\pi_{k}^{*}=\frac{1}{2}$.

Proof. Straight from 4.2 , with $\pi_{k}=1-\sum_{i=1}^{k-1} \pi_{i}$, then the first partial derivatives of $\phi(\Pi)$ are obtained as

$$
\frac{\partial \phi(\Pi)}{\partial \pi_{j}}=\left(\delta_{j}^{2}-\delta_{k}^{2}\right)-2\left(\delta_{j}-\delta_{k}\right) \sum_{i=1}^{k} \pi_{i} \delta_{i}, \quad \forall j=1,2, \ldots, k-1 .
$$


The value of $\pi_{1}$ which maximize $\phi(\Pi)$ can be found by solving $\frac{\partial \phi(\Pi)}{\partial \pi_{1}}=0$, implies

$$
2 \sum_{i=1}^{k} \pi_{i} \delta_{i}=\left(\delta_{1}+\delta_{k}\right)
$$

From 4.5 in 4.4, we get

$$
\frac{\partial \phi(\Pi)}{\partial \pi_{j}}=\left(\delta_{j}-\delta_{k}\right)\left(\delta_{j}-\delta_{1}\right), \quad \forall j=2,3, \ldots, k-1 .
$$

Since $\delta_{k}<\delta_{k-1}<\ldots<\delta_{1}=1$, then $\left(\delta_{j}-\delta_{k}\right)>0, \forall j=2,3, \ldots, k-1$, and $\left(\delta_{j}-\delta_{1}\right)<0, \forall j=2,3, \ldots, k-1$.

Thus, $\frac{\partial \phi(\Pi)}{\partial \pi_{j}}<0, \forall j=2,3, \ldots, k-1$. Therefore, $\phi(\Pi)$ is monotonically decreasing in $\pi_{j}$ for $j=2,3, \ldots, k-1$. Since $0 \leq \pi_{j} \leq 1$, then $\phi(\Pi)$ is maximized at $\pi_{j}^{*}=0, \forall j=2,3, \ldots, k-1$. As a result, the only nonzero $\pi_{j}$ for $j=1,2, \ldots, k-1$ is $\pi_{1}$.

Now, from 4.5 with $\pi_{j}=0, \forall j=2,3, \ldots, k-1$, and $\pi_{k}=1-\sum_{i=1}^{k-1} \pi_{i}$, then

$$
\left(\delta_{1}-\delta_{k}\right)\left(1-2 \pi_{1}\right)=0
$$

then $\pi_{1}^{*}=\frac{1}{2}$, and consequently $\pi_{k}^{*}=\frac{1}{2}$.

\subsection{A-optimality}

In this subsection, we consider another optimality criterion which depends on the trace of the variance-covariance matrix of the MLEs. The A-optimality criterion gives an overall measure of the total variance of the parameter estimates. The A-optimal allocation proportions $\pi_{1}^{*}, \pi_{2}^{*}, \ldots, \pi_{k}^{*}$ can be obtained by minimizing the objective function defined by

$$
\begin{aligned}
\varphi(\Pi) & =n \operatorname{tr}\left(\mathbf{F}^{-1}\left(\alpha, b, \theta_{1}\right)\right) \\
& =\frac{(b+1)^{2}+\theta_{1}^{2}\left(\sum_{i=1}^{k} \pi_{i} \delta_{i}\right)^{2}}{2 \alpha^{2}\left[\sum_{i=1}^{k} \pi_{i} \delta_{i}^{2}-\left(\sum_{i=1}^{k} \pi_{i} \delta_{i}\right)^{2}\right]}+\frac{8 \alpha^{4}+\theta_{1}^{2}\left(2(2-\log (2)-\gamma)^{2}+\pi^{2}-4\right)}{2 \alpha^{2}\left(\pi^{2}-4\right)} .
\end{aligned}
$$

For a multiple ramp-stress ALT, minimizing $\varphi(\Pi)$ in 4.7 is equivalent to selecting $\pi_{1}, \pi_{2}, \ldots, \pi_{k}$ for minimizing

$$
\eta(\Pi)=\left[\frac{(b+1)^{2}+\theta_{1}^{2}\left(\sum_{i=1}^{k} \pi_{i} \delta_{i}\right)^{2}}{\sum_{i=1}^{k} \pi_{i} \delta_{i}^{2}-\left(\sum_{i=1}^{k} \pi_{i} \delta_{i}\right)^{2}}\right] .
$$

Theorem 4.3. The A-optimal allocation proportions of a simple ramp-stress ALT for GHN distribution under complete sampling are

$$
\pi_{1}^{*}=\frac{(b+1)^{2}+\theta_{1}^{2} \delta_{2}^{2} \pm \sqrt{\left((b+1)^{2}+\theta_{1}^{2}\right)\left((b+1)^{2}+\theta_{1}^{2} \delta_{2}^{2}\right)}}{\theta_{1}^{2}\left(\delta_{2}^{2}-1\right)}, \text { and } \pi_{2}^{*}=1-\pi_{1}^{*} .
$$

Proof. In the case of a simple ramp-stress ALT, $k=2$ and $\pi_{2}=1-\pi_{1}$, then the objective function in 4.8 becomes

$$
\eta\left(\pi_{1}\right)=\left[\frac{(b+1)^{2}+\theta_{1}^{2}\left(\left(1-\pi_{1}\right) \delta_{2}+\pi_{1}\right)^{2}}{\pi_{1}+\left(1-\pi_{1}\right) \delta_{2}^{2}-\left(\left(1-\pi_{1}\right) \delta_{2}+\pi_{1}\right)^{2}}\right]
$$

to minimize $\eta\left(\pi_{1}\right)$ with respect to $\pi_{1}$, solving $\frac{d \eta\left(\pi_{1}\right)}{d \pi_{1}}=0$, gives

$$
\pi_{1}^{*}=\frac{(b+1)^{2}+\theta_{1}^{2} \delta_{2}^{2} \pm \sqrt{\left((b+1)^{2}+\theta_{1}^{2}\right)\left((b+1)^{2}+\theta_{1}^{2} \delta_{2}^{2}\right)}}{\theta_{1}^{2}\left(\delta_{2}^{2}-1\right)}, \text { and consequently } \pi_{2}^{*}=1-\pi_{1}^{*} .
$$


Theorem 4.4. The A-optimal allocation proportions of multiple ramp-stress ALT for GHN distribution under complete sampling are

$$
\pi_{1}^{*}=\frac{(b+1)^{2}+\theta_{1}^{2} \delta_{k}^{2} \pm \sqrt{\left((b+1)^{2}+\theta_{1}^{2}\right)\left((b+1)^{2}+\theta_{1}^{2} \delta_{k}^{2}\right)}}{\theta_{1}^{2}\left(\delta_{k}^{2}-1\right)}, \pi_{i}^{*}=0, \forall i=2,3, \ldots, k-1, \text { and } \pi_{k}^{*}=1-\pi_{1}^{*} .
$$

Proof. From the objective function in 4.8 , with $\pi_{k}=1-\sum_{i=1}^{k-1} \pi_{i}$, then

$$
\frac{\partial \eta(\Pi)}{\partial \pi_{j}}=\frac{2\left(\theta_{1}^{2} \sum_{i=1}^{k} \pi_{i} \delta_{i}^{2}+(b+1)^{2}\right)\left(\sum_{i=1}^{k} \pi_{i} \delta_{i}\right)\left(\delta_{j}-\delta_{k}\right)-\left(\delta_{j}^{2}-\delta_{k}^{2}\right)\left((b+1)^{2}+\theta_{1}^{2}\left(\sum_{i=1}^{k} \pi_{i} \delta_{i}\right)^{2}\right)}{\left(\sum_{i=1}^{k} \pi_{i} \delta_{i}^{2}-\left(\sum_{i=1}^{k} \pi_{i} \delta_{i}\right)^{2}\right)^{2}}, \begin{aligned}
& j=1,2, \ldots, k-1 .
\end{aligned}
$$

To get the value of $\pi_{1}$ which minimize $\eta(\Pi)$, solving $\frac{\partial \eta(\Pi)}{\partial \pi_{1}}=0$, implies

$$
2\left((b+1)^{2}+\theta_{1}^{2} \sum_{i=1}^{k} \pi_{i} \delta_{i}^{2}\right)\left(\sum_{i=1}^{k} \pi_{i} \delta_{i}\right)=\left(\delta_{1}+\delta_{k}\right)\left((b+1)^{2}+\theta_{1}^{2}\left(\sum_{i=1}^{k} \pi_{i} \delta_{i}\right)^{2}\right) .
$$

From (4.11) in 4.10), then

$$
\frac{\partial \eta(\Pi)}{\partial \pi_{j}}=\frac{\left(\delta_{j}-\delta_{k}\right)\left(\delta_{1}-\delta_{j}\right)\left((b+1)^{2}+\theta_{1}^{2}\left(\sum_{i=1}^{k} \pi_{i} \delta_{i}\right)^{2}\right)}{\left(\sum_{i=1}^{k} \pi_{i} \delta_{i}^{2}-\left(\sum_{i=1}^{k} \pi_{i} \delta_{i}\right)^{2}\right)^{2}}, \quad \forall j=2,3, \ldots, k-1 .
$$

Since $\delta_{k}<\delta_{k-1}<\ldots<\delta_{1}=1$, then $\left(\delta_{j}-\delta_{k}\right)>0, \forall j=2,3, \ldots, k-1$, and $\left(\delta_{1}-\delta_{j}\right)>0, \forall j=2,3, \ldots, k-1$.

Thus, $\frac{\partial \eta(\Pi)}{\partial \pi_{j}}>0, \forall j=2,3, \ldots, k-1$. Therefore, $\eta(\Pi)$ is monotonically increasing in $\pi_{j}$ for $j=2,3, \ldots, k-1$. Because of $0 \leq \pi_{j} \leq 1$, then $\eta(\Pi)$ is minimized at $\pi_{j}^{*}=0, \forall j=2,3, \ldots, k-1$. As a result, the only nonzero $\pi_{j}$ for $j=1,2, \ldots, k-1$ is $\pi_{1}$.

Now, from 4.11 with $\pi_{j}=0, \forall j=2,3, \ldots, k-1$ and $\pi_{k}=1-\sum_{i=1}^{k-1} \pi_{i}$, then

$$
2\left((b+1)^{2}+\theta_{1}^{2}\left(\pi_{1}+\delta_{k}^{2}\left(1-\pi_{1}\right)\right)\right)\left(\pi_{1}+\delta_{k}\left(1-\pi_{1}\right)\right)=\left(1+\delta_{k}\right)\left((b+1)^{2}+\theta_{1}^{2}\left(\pi_{1}+\delta_{k}\left(1-\pi_{1}\right)\right)^{2}\right),
$$

then

$$
\pi_{1}^{*}=\frac{(b+1)^{2}+\theta_{1}^{2} \delta_{k}^{2} \pm \sqrt{\left((b+1)^{2}+\theta_{1}^{2}\right)\left((b+1)^{2}+\theta_{1}^{2} \delta_{k}^{2}\right)}}{\theta_{1}^{2}\left(\delta_{k}^{2}-1\right)}, \text { and consequently } \pi_{k}^{*}=1-\pi_{1}^{*} .
$$

From Theorems 4.2 and 4.4 we observed the following:

1. From Theorems 4.2 and 4.4 we observed that the optimal multiple ramp-stress ALT is degenerate to simple ramp-stress ALT under D and A-optimality criteria.

2. From Theorem 4.2, we noted that the D-optimality allocates an equal number of test units at the lowest stress level $S_{1}(t)$ and the highest stress level $S_{k}(t)$. 


\section{Application}

In this section, the theoretical results developed in Sections 3 and 4 are illustrated with a real data example. Moreover, this example is used to show that the GHN distribution can be a possible alternative to gamma, Weibull and exponentiated exponential distributions.

The lifetime data in Table 5.1 from Zhu 38 were collected from ramp-voltage tests of miniature light bulbs. In this test, 62 miniature light bulbs were tested under ramp-rate $2.01 \mathrm{~V} / \mathrm{h}$, and 61 miniature light bulbs were tested under ramp-rate $2.015 \mathrm{~V} / \mathrm{h}$.

To check the validity of GHN, gamma, Weibull and exponentiated exponential distributions with the data in Table 5.1 for each ramp-stress $S_{i}(t), i=1,2$. We compute Kolmogorov-Smirnov (K-S) distance between the empirical distribution function and the fitted distribution function when the parameters are obtained by MLE. The values of K-S distance and the corresponding P-values for each stress level are presented in Table 5.2 It is clear that the estimated GHN, gamma, Weibull and exponentiated exponential distributions provide a good fit to the given data due to all P-values are greater than 0.05.

Akaike information criterion (AIC) is used for the purpose of comparison between the four models. For this reason, the method of maximum likelihood is used to obtain the estimates of the parameters of the four distributions. The MLEs of the parameters $\alpha, b$ and $\theta_{1}$, and AIC for the four distributions are summarized in Table 5.3 Since the four models have the same number of parameters, it follows that the GHN distribution provides a better fit compared to gamma, Weibull and exponentiated exponential distributions regarding AIC.

Table 5.1. The lifetime data from ramp-voltage tests

\begin{tabular}{|c|c|c|c|c|c|c|c|c|c|c|c|}
\hline \multicolumn{6}{|c|}{ Ramp-Rate $2.01 \mathrm{~V} / \mathrm{h}$} & \multicolumn{6}{|c|}{ Ramp-Rate $2.015 \mathrm{~V} / \mathrm{h}$} \\
\hline No. & Failure Time & No. & Failure Time & No. & Failure Time & No. & Failure Time & No. & Failure Time & No. & Failure Time \\
\hline 1 & 13.57 & 22 & 72.33 & 43 & 42.06 & 1 & 19.3 & 22 & 49.65 & 43 & 31.00 \\
\hline 2 & 19.92 & 23 & 72.60 & 44 & 47.88 & 2 & 23.28 & 23 & 51.42 & 44 & 34.81 \\
\hline 3 & 23.3 & 24 & 75.43 & 45 & 54.21 & 3 & 23.50 & 24 & 51.27 & 45 & 36.03 \\
\hline 4 & 27.81 & 25 & 75.85 & 46 & 54.55 & 4 & 26.50 & 25 & 53.25 & 46 & 43.08 \\
\hline 5 & 31.16 & 26 & 76.20 & 47 & 55.85 & 5 & 27.42 & 26 & 54.25 & 47 & 45.63 \\
\hline 6 & 31.56 & 27 & 77.78 & 48 & 56.43 & 6 & 28.32 & 27 & 55.47 & 48 & 46.03 \\
\hline 7 & 34.00 & 28 & 79.13 & 49 & 58.86 & 7 & 28.62 & 28 & 56.83 & 49 & 46.33 \\
\hline 8 & 46.26 & 29 & 80.65 & 50 & 60.60 & 8 & 30.62 & 29 & 56.17 & 50 & 49.62 \\
\hline 9 & 46.41 & 30 & 82.65 & 51 & 62.48 & 9 & 34.42 & 30 & 8.85 & 51 & 49.86 \\
\hline 10 & 50.60 & 31 & 90.33 & 52 & 62.81 & 10 & 35.30 & 31 & 11.31 & 52 & 50.66 \\
\hline 11 & 56.76 & 32 & 14.51 & 53 & 63.41 & 11 & 35.48 & 32 & 11.83 & 53 & 50.93 \\
\hline 12 & 56.85 & 33 & 15.61 & 54 & 63.76 & 12 & 38.30 & 33 & 14.50 & 54 & 51.03 \\
\hline 13 & 60.13 & 34 & 15.85 & 55 & 64.18 & 13 & 40.52 & 34 & 14.83 & 55 & 51.73 \\
\hline 14 & 65.00 & 35 & 17.73 & 56 & 66.15 & 14 & 43.83 & 35 & 17.73 & 56 & 51.95 \\
\hline 15 & 65.86 & 36 & 19.65 & 57 & 66.41 & 15 & 43.00 & 36 & 19.35 & 57 & 52.36 \\
\hline 16 & 66.20 & 37 & 21.05 & 58 & 69.91 & 16 & 43.00 & 37 & 25.50 & 58 & 54.78 \\
\hline 17 & 66.40 & 38 & 21.20 & 59 & 71.73 & 17 & 43.12 & 38 & 26.15 & 59 & 55.58 \\
\hline 18 & 66.80 & 39 & 24.21 & 60 & 72.46 & 18 & 44.43 & 39 & 27.45 & 60 & 55.83 \\
\hline 19 & 66.93 & 40 & 24.85 & 61 & 73.78 & 19 & 45.32 & 40 & 27.61 & 61 & $5 \% .13$ \\
\hline 20 & 68.25 & 41 & 31.18 & 62 & 78.91 & 20 & 47.58 & 41 & 28.05 & & \\
\hline 21 & 70.23 & 42 & 35.08 & & & 21 & 47.65 & 42 & 30.96 & & \\
\hline
\end{tabular}

Table 5.2. $K$-S distances and the corresponding P-values of each stress level for GHN, gamma, Weibull and exponentiated exponential distributions

\begin{tabular}{ccccccccc}
\hline & \multicolumn{2}{c}{$G H N$} & \multicolumn{2}{c}{ gamma } & \multicolumn{2}{c}{ Weibull } & \multicolumn{2}{c}{ exponentiated exponential } \\
\hline Ramp-Rate & $2.01 \mathrm{~V} / \mathrm{h}$ & $2.015 \mathrm{~V} / \mathrm{h}$ & $2.01 \mathrm{~V} / \mathrm{h}$ & $2.015 \mathrm{~V} / \mathrm{h}$ & $2.01 \mathrm{~V} / \mathrm{h}$ & $2.015 \mathrm{~V} / \mathrm{h}$ & $2.01 \mathrm{~V} / \mathrm{h}$ & $2.015 \mathrm{~V} / \mathrm{h}$ \\
\hline K-S distance & 0.1290 & 0.1311 & 0.1612 & 0.1639 & 0.1290 & 0.1803 & 0.1774 & 0.2131 \\
\hline P-value & 0.6846 & 0.6748 & 0.3980 & 0.3879 & 0.6846 & 0.2747 & 0.2849 & 0.1254 \\
\hline
\end{tabular}

Table 5.3. AIC and estimated parameters for GHN, gamma, Weibull and exponentiated exponential distributions

\begin{tabular}{ccccc}
\hline \multirow{2}{*}{ Distribution } & \multirow{2}{*}{ AIC } & \multicolumn{3}{c}{ Estimated parameters } \\
\cline { 3 - 5 } & & $\hat{\alpha}$ & $\hat{b}$ & $\hat{\theta}_{1}$ \\
\hline GHN & 980.19 & 2.6268 & 134.053 & 0.4699 \\
\hline gamma & 1071.74 & 5.2859 & 123.329 & 0.0801 \\
\hline Weibull & 1048.84 & 3.0831 & 131.889 & 0.4486 \\
\hline exponentiated exponential & 1082.41 & 6.0945 & 118.33 & 0.1813 \\
\hline
\end{tabular}



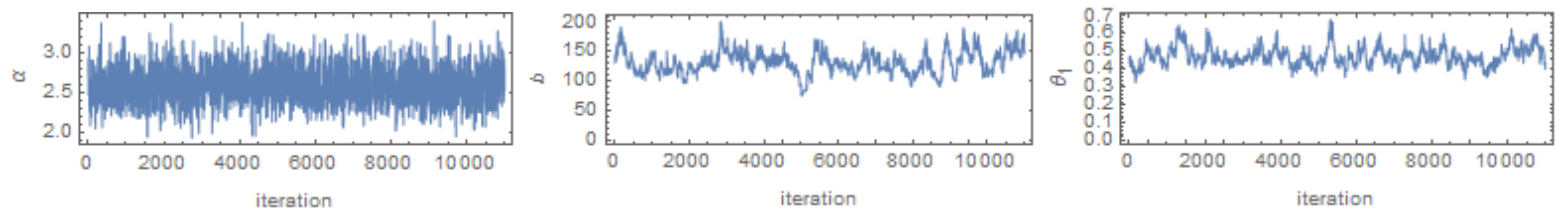

Figure 1: MCMC trace plots of simulated samples for $\alpha, b$ and $\theta_{1}$.
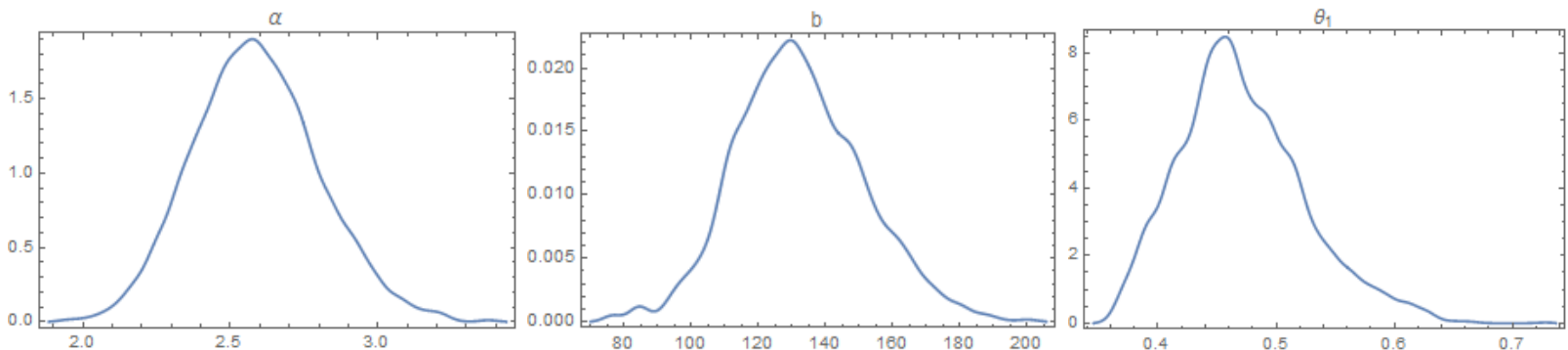

Figure 2: Kernel densities estimate of $\alpha, b$ and $\theta_{1}$.

The MLEs, LSEs, MEs, and BEs under SE and LINEX loss functions of the parameters $\alpha, b$ and $\theta_{1}$ are introduced in Table 5.4. For this data set, Bayesian analysis is conducted in case of NIPs. Table 5.5 includes $99 \%$ and $95 \%$ NACIs and BCIs of the parameters $\alpha, b$ and $\theta_{1}$. From the results in Table 5.5, we note that the BEs of $b$ and $\theta_{1}$ give more accurate results than the MLEs through the length of the intervals while the MLE of $\alpha$ more accurate than the BE of $\alpha$. The MCMC iterations of $\alpha, b$ and $\theta_{1}$ are plotted in Figure 1 Trace plots indicate that the MCMC samples are well mixed and stationary achieved. Kernel densities estimate of $\alpha, b$ and $\theta_{1}$ are plotted in Figure 2. This figure indicates that the marginal posterior distributions of $\alpha, b$ and $\theta_{1}$ are symmetric.

Table 5.4. MLEs, LSEs, MEs, and BEs under SE (BSE) and LINEX (BLINEX) loss functions of $\alpha, b$ and $\theta_{1}$ for rampvoltage tests

\begin{tabular}{ccccccc}
\hline$\vartheta$ & $M L E$ & $L S E$ & $M E$ & $B S E$ & $B L I N E X(c=3)$ & $B L I N E X(c=-3)$ \\
\hline$\hat{\alpha}$ & 2.6268 & 0.823987 & 2.2881 & 2.5992 & 2.5326 & 2.6715 \\
$\hat{b}$ & 134.053 & 112.503 & 78.6234 & 132.346 & 96.442 & 154.548 \\
$\hat{\theta_{1}}$ & 0.4699 & 0.5509 & 0.7578 & 0.4811 & 0.4777 & 0.48474 \\
\hline
\end{tabular}

Table 5.5. $99 \%$ and $95 \%$ NACIs and BCIs for $\alpha, b$ and $\theta_{1}$ for ramp-voltage tests

\begin{tabular}{ccccc}
\hline$\vartheta$ & \multicolumn{2}{c}{$99 \%$ NACI and $B C I$} & \multicolumn{2}{c}{$95 \%$ NACI and $B C I$} \\
\cline { 2 - 5 } & $N A C I$ & $B C I$ & $N A C I$ & $B C I$ \\
\hline$\alpha$ & $(2.0820,3.1716)$ & $(2.0752,3.2134)$ & $(2.2130,3.0407)$ & $(2.1964,3.0500)$ \\
$b$ & $(83.6364,184.471)$ & $(101.062,178.988)$ & $(95.7521,172.355)$ & $(105.131,166.873)$ \\
$\theta_{1}$ & $(0.3205,0.6192)$ & $(0.3744,0.6032)$ & $(0.3564,0.5833)$ & $(0.3884,0.5765)$ \\
\hline
\end{tabular}

Based on the MLEs $\left(\hat{\alpha}=2.6268, \hat{b}=134.053\right.$ and $\left.\hat{\theta_{1}}=0.4699\right)$, the optimal allocation proportions can be found by employing the methods described in Section 4. For the D-optimality, the optimal allocation is found to be $(0.50,0.50)$. For the A-optimality, the optimal allocation is found to be $(0.50,0.50)$.

\section{Simulation study}

In this section, based on Monte Carlo simulations, a numerical study is conducted in order to compare the performance of the estimation methods. The numerical results are carried out based on 1,000 different samples of different sizes generated from $\mathrm{CDF}$ in 2.3). The population parameters values used in the simulation study are $\alpha=1.1, b=0.66$ and $\theta_{1}=0.6$ 
with two settings of stress rate factor. The first setting is $0.1=\psi_{k}<\psi_{k-1}<\ldots<\psi_{2}<\psi_{1}=1$, and the second setting is $0.01=\psi_{k}<\psi_{k-1}<\ldots<\psi_{2}<\psi_{1}=1$. The BEs of the model parameters under SE and LINEX loss functions are obtained using algorithm (1), with $N=11000, M=1000$. Tables 6.1 and 6.3 introduce the average of the MEs, LSEs and MLEs of the parameters $\alpha, b$ and $\theta_{1}$ with their mean square errors (MSEs). Tables 6.2 and 6.4 give the average of the BEs under SE and LINEX loss functions with their MSEs. While, Tables 6.5 and 6.6 include the 95\% NACIs, BCIs, and their lengths and the coverage probabilities of the model parameters.

Table 6.1. The average of MEs, LSEs and MLEs of $\left(\alpha, b, \theta_{1}\right)$ with their MSEs. The population parameters values are $(\alpha=1.1, b=0.66$ and $\left.\theta_{1}=0.6\right)$, values of the prior parameters $\left(\mu_{1}=12.1, \mu_{2}=4.356, \mu_{3}=3.6 \lambda_{1}=11, \lambda_{2}=6.6\right.$ and $\left.\lambda_{3}=6\right)$ and $\psi_{k}=0.1$.

\begin{tabular}{|c|c|c|c|c|c|c|c|c|c|}
\hline \multirow{4}{*}{$k$} & \multirow{4}{*}{$n$} & \multirow{4}{*}{ criterion } & \multirow{4}{*}{$n_{i}$} & \multicolumn{2}{|c|}{$M E s$} & \multicolumn{2}{|c|}{ LSES } & \multicolumn{2}{|c|}{$M L E s$} \\
\hline & & & & $\check{\alpha}$ & $M S E(\check{\alpha})$ & $\breve{\alpha}$ & $M S E(\breve{\alpha})$ & $\hat{\alpha}$ & $M S E(\hat{\alpha})$ \\
\hline & & & & $\check{b}$ & $M S E(\check{b})$ & $\breve{b}$ & $M S E(\breve{b})$ & $\hat{b}$ & $M S E(\hat{b})$ \\
\hline & & & & $\check{\theta_{1}}$ & $M S E\left(\check{\theta_{1}}\right)$ & $\breve{\theta_{1}}$ & $M S E\left(\breve{\theta_{1}}\right)$ & $\hat{\theta_{1}}$ & $M S E\left(\hat{\theta_{1}}\right)$ \\
\hline \multirow{3}{*}{4} & & & $20, \quad i=1$ & 0.9475 & 0.1690 & 0.3100 & 0.6286 & 1.1594 & 0.0308 \\
\hline & 40 & $D$ & $0, \quad i=2,3$ & 0.3865 & 0.1925 & 0.6659 & 0.0170 & 0.6576 & 0.0070 \\
\hline & & & $20, \quad i=4$ & 0.5484 & 0.0119 & 0.6280 & 0.0111 & 0.5918 & 0.0050 \\
\hline \multirow{3}{*}{4} & & & $23, \quad i=1$ & 0.9256 & 0.1535 & 0.3175 & 0.6168 & 1.1796 & 0.0336 \\
\hline & 40 & $A$ & $0, \quad i=2,3$ & 0.3686 & 0.1971 & 0.6389 & 0.0172 & 0.6519 & 0.0066 \\
\hline & & & $17, \quad i=4$ & 0.5501 & 0.0114 & $0.610^{7}$ & 0.0078 & 0.5845 & 0.0044 \\
\hline \multirow{3}{*}{4} & & & $40, \quad i=1$ & 0.9667 & 0.1257 & 0.3113 & 0.6248 & 1.1339 & 0.0130 \\
\hline & 80 & $D$ & $0, \quad i=2,3$ & 0.4216 & 0.1678 & 0.6653 & 0.0098 & 0.671 & 0.0035 \\
\hline & & & $40, \quad i=4$ & 0.5612 & 0.0070 & 0.6202 & 0.0058 & 0.6075 & 0.0019 \\
\hline \multirow{3}{*}{4} & & & $46, \quad i=1$ & 0.9572 & 0.1171 & 0.3131 & 0.6221 & 1.1259 & 0.0117 \\
\hline & 80 & $A$ & $0, \quad i=2,3$ & 0.4287 & 0.1586 & 0.6761 & 0.0087 & 0.6824 & 0.0037 \\
\hline & & & $34, \quad i=4$ & 0.5595 & 0.0073 & 0.6309 & 0.0067 & 0.6201 & 0.0024 \\
\hline \multirow{3}{*}{4} & & & $60, \quad i=1$ & 0.9551 & 0.1109 & 0.3117 & 0.6232 & 1.1118 & 0.0067 \\
\hline & 120 & $D$ & $0, \quad i=2,3$ & 0.4258 & 0.1574 & 0.6764 & 0.0058 & 0.6777 & 0.0024 \\
\hline & & & $60, \quad i=4$ & 0.5648 & 0.0059 & 0.6205 & 0.0031 & 0.6173 & 0.0013 \\
\hline \multirow{3}{*}{4} & & & $69, \quad i=1$ & 0.9772 & 0.0995 & 0.3119 & 0.6228 & 1.1166 & 0.0077 \\
\hline & 120 & $A$ & $0, \quad i=2,3$ & 0.4541 & 0.1421 & 0.6798 & 0.0067 & 0.6863 & 0.0040 \\
\hline & & & $51, \quad i=4$ & 0.5679 & 0.0058 & 0.6237 & 0.0039 & 0.6217 & 0.0026 \\
\hline
\end{tabular}

Table 6.2. The average of BEs under SE (BSE) and LINEX (BLINEX) loss functions of ( $\left.\alpha, b, \theta_{1}\right)$ with their MSEs. The population parameters values are $\left(\alpha=1.1, b=0.66\right.$ and $\left.\theta_{1}=0.6\right)$, values of the prior parameters $\left(\mu_{1}=12.1, \mu_{2}=4.356, \mu_{3}=3.6 \lambda_{1}=11\right.$, $\lambda_{2}=6.6$ and $\left.\lambda_{3}=6\right)$ and $\psi_{k}=0.1$.

\begin{tabular}{|c|c|c|c|c|c|c|c|c|c|}
\hline \multirow{4}{*}{$k$} & \multirow{4}{*}{$n$} & \multirow{4}{*}{ criterion } & \multirow{4}{*}{$n_{i}$} & \multicolumn{2}{|c|}{$B S E$} & \multicolumn{2}{|c|}{$B L I N E X(c=-3)$} & \multicolumn{2}{|c|}{$B L I N E X(c=3)$} \\
\hline & & & & $\tilde{\alpha}$ & $M S E(\tilde{\alpha})$ & $\tilde{\alpha}$ & $M S E(\tilde{\alpha})$ & $\tilde{\alpha}$ & $M S E(\tilde{\alpha})$ \\
\hline & & & & $\tilde{b}$ & $M S E(\tilde{b})$ & $\tilde{b}$ & $M S E(\tilde{b})$ & $\tilde{b}$ & $M S E(\tilde{b})$ \\
\hline & & & & $\tilde{\theta_{1}}$ & $M S E\left(\tilde{\theta_{1}}\right)$ & $\tilde{\theta_{1}}$ & $M S E\left(\tilde{\theta_{1}}\right)$ & $\tilde{\theta_{1}}$ & $M S E\left(\tilde{\theta_{1}}\right)$ \\
\hline \multirow{3}{*}{4} & & & $20, \quad i=1$ & 1.1116 & 0.0162 & 1.1408 & 0.0196 & 1.0842 & 0.0147 \\
\hline & 40 & $D$ & $0, \quad i=2,3$ & 0.6472 & 0.0062 & 0.6591 & 0.0060 & 0.6353 & 0.0066 \\
\hline & & & $20, \quad i=4$ & 0.5988 & 0.0042 & 0.6067 & 0.0044 & 0.5913 & 0.0041 \\
\hline \multirow{3}{*}{4} & & & $23, \quad i=1$ & 1.1274 & 0.0167 & 1.1573 & 0.0211 & 1.0993 & 0.0143 \\
\hline & 40 & $A$ & $0, \quad i=2,3$ & 0.6390 & 0.0060 & 0.6510 & 0.0057 & 0.6270 & 0.0067 \\
\hline & & & $17, \quad i=4$ & 0.5913 & 0.00371 & 0.5982 & 0.00374 & 0.5848 & 0.00377 \\
\hline \multirow{3}{*}{4} & & & $40, \quad i=1$ & 1.0996 & 0.00246 & 1.1074 & 0.00259 & 1.0920 & 0.00244 \\
\hline & 80 & $D$ & $0, \quad i=2,3$ & 0.6646 & 0.00178 & 0.6687 & 0.00185 & 0.6604 & 0.00174 \\
\hline & & & $40, \quad i=4$ & 0.6066 & 0.00105 & 0.6095 & 0.00111 & 0.6037 & 0.00100 \\
\hline \multirow{3}{*}{4} & & & $46, \quad i=1$ & 1.0951 & 0.00231 & 1.1027 & 0.00237 & 1.0876 & 0.00238 \\
\hline & 80 & $A$ & $0, \quad i=2,3$ & 0.6711 & 0.00174 & 0.6754 & 0.00187 & 0.6667 & 0.00165 \\
\hline & & & $34, \quad i=4$ & 0.6170 & 0.00139 & 0.6198 & 0.00151 & 0.6141 & 0.00129 \\
\hline \multirow{3}{*}{4} & & & $60, \quad i=1$ & 1.0899 & 0.00020 & 1.0912 & 0.00018 & 1.0886 & 0.00023 \\
\hline & 120 & $D$ & $0, \quad i=2,3$ & 0.6632 & 0.00020 & 0.6643 & 0.00021 & 0.6622 & 0.00019 \\
\hline & & & $60, \quad i=4$ & 0.6056 & 0.00018 & 0.6065 & 0.00019 & 0.6047 & 0.00017 \\
\hline \multirow{3}{*}{4} & & & $69, \quad i=1$ & 1.0896 & 0.00022 & 1.0909 & 0.00020 & 1.0884 & 0.00025 \\
\hline & 120 & $A$ & $0, \quad i=2,3$ & 0.6658 & 0.00027 & 0.6669 & 0.00029 & 0.6647 & 0.00026 \\
\hline & & & $51, \quad i=4$ & 0.6074 & 0.00038 & 0.6082 & 0.00039 & 0.6065 & 0.00037 \\
\hline
\end{tabular}


Table 6.3. The average of MEs, LSEs and MLEs of $\left(\alpha, b, \theta_{1}\right)$ with their MSEs. The population parameters values are $(\alpha=1.1, b=0.66$ and $\left.\theta_{1}=0.6\right)$, values of the prior parameters $\left(\mu_{1}=12.1, \mu_{2}=4.356, \mu_{3}=3.6 \lambda_{1}=11, \lambda_{2}=6.6\right.$ and $\left.\lambda_{3}=6\right)$ and $\psi_{k}=0.01$.

\begin{tabular}{|c|c|c|c|c|c|c|c|c|c|}
\hline \multirow{4}{*}{$k$} & \multirow{4}{*}{$n$} & \multirow{4}{*}{ criterion } & \multirow{4}{*}{$n_{i}$} & \multicolumn{2}{|c|}{$M E s$} & \multicolumn{2}{|c|}{$L S E S$} & \multicolumn{2}{|c|}{$M L E s$} \\
\hline & & & & $\check{\alpha}$ & $M S E(\check{\alpha})$ & $\breve{\alpha}$ & $M S E(\breve{\alpha})$ & $\hat{\alpha}$ & $M S E(\hat{\alpha})$ \\
\hline & & & & $\check{b}$ & $M S E(\breve{b})$ & $\breve{b}$ & $M S E(\breve{b})$ & $\hat{b}$ & $M S E(\hat{b})$ \\
\hline & & & & $\check{\theta_{1}}$ & $M S E\left(\ddot{\theta_{1}}\right)$ & $\breve{\theta_{1}}$ & $M S E\left(\breve{\theta_{1}}\right)$ & $\hat{\theta_{1}}$ & $M S E\left(\hat{\theta_{1}}\right)$ \\
\hline \multirow{3}{*}{4} & & & $20, \quad i=1$ & 1.2574 & 0.1036 & 0.3127 & 0.6244 & 1.1590 & 0.0268 \\
\hline & 40 & $D$ & $0, \quad i=2,3$ & 0.8036 & 0.1338 & 0.6626 & 0.0048 & 0.6613 & 0.0019 \\
\hline & & & $20, \quad i=4$ & 0.5565 & 0.0110 & 0.0164 & 0.0540 & 0.5968 & 0.0059 \\
\hline \multirow{3}{*}{4} & & & $28, \quad i=1$ & 1.2281 & 0.0700 & 0.3141 & 0.6223 & 1.1602 & 0.0269 \\
\hline & 40 & $A$ & $0, \quad i=2,3$ & 0.8060 & 0.1317 & 0.6629 & 0.0052 & 0.6670 & 0.0023 \\
\hline & & & $12, \quad i=4$ & 0.5611 & 0.0087 & 0.6248 & 0.0098 & 0.5994 & 0.0049 \\
\hline \multirow{3}{*}{4} & & & $40, \quad i=1$ & 1.1858 & 0.0443 & 0.3137 & 0.6207 & 1.1320 & 0.0121 \\
\hline & 80 & $D$ & $0, \quad i=2,3$ & 0.7345 & 0.0531 & 0.6616 & 0.0024 & 0.6619 & 0.00099 \\
\hline & & & $40, \quad i=4$ & 0.5832 & 0.0042 & 0.6135 & 0.0076 & 0.6023 & 0.0031 \\
\hline \multirow{3}{*}{4} & & & $57, \quad i=1$ & 1.1563 & 0.0290 & 0.3144 & 0.6197 & 1.1343 & 0.0131 \\
\hline & 80 & $A$ & $0, \quad i=2,3$ & 0.7129 & 0.0424 & 0.6604 & 0.0028 & 0.6636 & 0.0013 \\
\hline & & & $23, \quad i=4$ & 0.5869 & 0.0036 & 0.6079 & 0.0040 & 0.5998 & 0.0021 \\
\hline \multirow{3}{*}{4} & & & $60, \quad i=1$ & $1.16^{71}$ & 0.0310 & 0.3122 & 0.6226 & 1.1216 & 0.0087 \\
\hline & 120 & $D$ & $0, \quad i=2,3$ & 0.7055 & 0.0294 & 0.6630 & 0.0016 & 0.6646 & 0.00061 \\
\hline & & & $60, \quad i=4$ & 0.5860 & 0.0027 & 0.6144 & 0.0049 & 0.6099 & 0.0019 \\
\hline \multirow{3}{*}{4} & & & $85, \quad i=1$ & 1.1450 & 0.0232 & 0.3093 & 0.6268 & $1.116^{r}$ & 0.0082 \\
\hline & 120 & $A$ & $0, \quad i=2,3$ & 0.7028 & 0.0324 & 0.6563 & 0.0019 & 0.6609 & 0.00072 \\
\hline & & & $35, \quad i=4$ & 0.5881 & 0.0020 & 0.6020 & 0.0033 & 0.5987 & 0.0015 \\
\hline
\end{tabular}

Table 6.4. The average of BEs under SE (BSE) and LINEX (BLINEX) loss functions of $\left(\alpha, b, \theta_{1}\right)$ with their MSEs. The population parameters values are $\left(\alpha=1.1, b=0.66\right.$ and $\left.\theta_{1}=0.6\right)$, values of the prior parameters $\left(\mu_{1}=12.1, \mu_{2}=4.356\right.$, $\mu_{3}=3.6 \lambda_{1}=11, \lambda_{2}=6.6$ and $\left.\lambda_{3}=6\right)$ and $\psi_{k}=0.01$.

\begin{tabular}{|c|c|c|c|c|c|c|c|c|c|}
\hline \multirow{4}{*}{$k$} & \multirow{4}{*}{$n$} & \multirow{4}{*}{ criterion } & \multirow{4}{*}{$n_{i}$} & \multicolumn{2}{|c|}{$B S E$} & \multicolumn{2}{|c|}{$B L I N E X(c=-3)$} & \multicolumn{2}{|c|}{$B L I N E X(c=3)$} \\
\hline & & & & $\tilde{\alpha}$ & $M S E(\tilde{\alpha})$ & $\tilde{\alpha}$ & $M S E(\tilde{\alpha})$ & $\tilde{\alpha}$ & $M S E(\tilde{\alpha})$ \\
\hline & & & & $\tilde{b}$ & $M S E(\tilde{b})$ & $\tilde{b}$ & $M S E(\tilde{b})$ & $\tilde{b}$ & $M S E(\tilde{b})$ \\
\hline & & & & $\tilde{\theta_{1}}$ & $M S E\left(\tilde{\theta_{1}}\right)$ & $\tilde{\theta_{1}}$ & $M S E\left(\tilde{\theta_{1}}\right)$ & $\tilde{\theta_{1}}$ & $M S E\left(\tilde{\theta_{1}}\right)$ \\
\hline \multirow{3}{*}{4} & & & $20, \quad i=1$ & 1.1111 & 0.0142 & 1.1400 & 0.0172 & 1.0840 & 0.0130 \\
\hline & 40 & $D$ & $0, \quad i=2,3$ & 0.6578 & 0.00181 & 0.6609 & 0.00181 & 0.6547 & 0.00183 \\
\hline & & & $20, \quad i=4$ & 0.6038 & 0.0051 & 0.6140 & 0.0055 & 0.5944 & 0.0048 \\
\hline \multirow{3}{*}{4} & & & $28, \quad i=1$ & 1.1114 & 0.0139 & 1.1404 & 0.0170 & 1.0842 & 0.0127 \\
\hline & 40 & $A$ & $0, \quad i=2,3$ & 0.6581 & 0.00219 & 0.662 & 0.00219 & 0.6541 & 0.0022 \\
\hline & & & $12, \quad i=4$ & 0.6048 & 0.0043 & 0.6124 & 0.0046 & 0.5976 & 0.00419 \\
\hline \multirow{3}{*}{4} & & & $40, \quad i=1$ & 1.0985 & 0.00231 & 1.1062 & 0.00242 & 1.0909 & 0.00232 \\
\hline & 80 & $D$ & $0, \quad i=2,3$ & 0.6600 & 0.00072 & 0.6613 & 0.00072 & 0.6587 & 0.00075 \\
\hline & & & $40, \quad i=4$ & 0.6025 & 0.00161 & 0.6060 & 0.00167 & 0.5990 & 0.00158 \\
\hline \multirow{3}{*}{4} & & & $57, \quad i=1$ & 1.0996 & 0.00248 & 1.1073 & 0.00262 & 1.0919 & 0.00247 \\
\hline & 80 & $A$ & $0, \quad i=2,3$ & 0.6596 & 0.00100 & 0.6612 & 0.00100 & 0.6580 & 0.00101 \\
\hline & & & $23, \quad i=4$ & 0.6006 & 0.00126 & 0.6034 & 0.00129 & 0.5979 & 0.00125 \\
\hline \multirow{3}{*}{4} & & & $60, \quad i=1$ & 1.0915 & 0.00019 & 1.0927 & 0.00017 & 1.0902 & 0.00021 \\
\hline & 120 & $D$ & $0, \quad i=2,3$ & 0.6612 & 0.00020 & 0.6617 & 0.00020 & 0.6607 & 0.00024 \\
\hline & & & $60, \quad i=4$ & 0.6024 & 0.00017 & 0.6033 & 0.00018 & 0.6015 & 0.00017 \\
\hline \multirow{3}{*}{4} & & & $85, \quad i=1$ & 1.0965 & 0.00251 & 1.1026 & 0.00257 & 1.0905 & 0.00253 \\
\hline & 120 & $A$ & $0, \quad i=2,3$ & 0.6583 & 0.00060 & 0.6594 & 0.00060 & 0.6571 & 0.00061 \\
\hline & & & $35, \quad i=4$ & 0.5999 & 0.00109 & 0.6019 & 0.00110 & 0.5979 & 0.00108 \\
\hline
\end{tabular}

Table 6.5. NACIs and BCIs of $\left(\alpha, b, \theta_{1}\right)$ with their lengths (LNACIs and LBCIs) and coverage probabilities (CPNACIs and CPBCIs). The population parameters values are $\left(\alpha=1.1, b=0.66\right.$ and $\left.\theta_{1}=0.6\right)$, values of the prior parameters $\left(\mu_{1}=12.1\right.$, $\mu_{2}=4.356, \mu_{3}=3.6 \lambda_{1}=11, \lambda_{2}=6.6$ and $\left.\lambda_{3}=6\right)$ and $\psi_{k}=0.1$. 


\begin{tabular}{|c|c|c|c|c|c|c|c|c|c|}
\hline \multirow{4}{*}{$k$} & \multirow{4}{*}{$n$} & \multirow{4}{*}{ criterion } & \multirow{4}{*}{$n_{i}$} & \multicolumn{3}{|c|}{ NACIs } & \multicolumn{3}{|c|}{$B C I s$} \\
\hline & & & & $N A C I(\alpha)$ & $L N A C I(\alpha)$ & $C P N A C I(\alpha)$ & $B C I(\alpha)$ & $L B C I(\alpha)$ & $C P B C I(\alpha)$ \\
\hline & & & & $N A C I(b)$ & $L N A C I(b)$ & $C P N A C I(b)$ & $B C I(b)$ & $L B C I(b)$ & $C P B C I(b)$ \\
\hline & & & & $N A C I\left(\theta_{1}\right)$ & $L N A C I\left(\theta_{1}\right)$ & $C P N B C I\left(\theta_{1}\right)$ & $B C I\left(\theta_{1}\right)$ & $\operatorname{LBCI}\left(\theta_{1}\right)$ & $C P B C I\left(\theta_{1}\right)$ \\
\hline \multirow{3}{*}{4} & & & $20, \quad i=1$ & $(0.85737,1.46155)$ & 0.6041 & 0.94 & $(0.85913,1.39224)$ & 0.5331 & 0.97 \\
\hline & 40 & $D$ & $0, \quad i=2,3$ & $(0.48969,0.82551)$ & 0.3358 & 0.94 & $(0.4723,0.82159)$ & 0.3492 & 0.97 \\
\hline & & & $20, \quad i=4$ & $(0.45757,0.72605)$ & 0.2684 & 0.91 & $(0.47213,0.75159)$ & 0.2794 & 0.95 \\
\hline \multirow{3}{*}{4} & & & $23, \quad i=1$ & $(0.87265,1.48673)$ & 0.6140 & 0.95 & $(0.87166,1.4116)$ & 0.5399 & 0.98 \\
\hline & 40 & $A$ & $0, \quad i=2,3$ & $(0.48521,0.81874)$ & 0.3335 & 0.96 & $(0.46035,0.81184)$ & 0.3514 & 0.97 \\
\hline & & & $17, \quad i=4$ & $(0.45969,0.70948)$ & 0.2497 & 0.92 & $(0.47167,0.73296)$ & 0.2612 & 0.95 \\
\hline \multirow{3}{*}{4} & & & $40, \quad i=1$ & $(0.9266,1.34131)$ & 0.4147 & 0.94 & $(0.96379,1.24364)$ & 0.2798 & 0.99 \\
\hline & 80 & $D$ & $0, \quad i=2,3$ & $(0.55102,0.79098)$ & 0.2399 & 0.96 & $(0.56146,0.76838)$ & 0.2069 & 0.99 \\
\hline & & & $40, \quad i=4$ & $(0.50826,0.70681)$ & 0.1985 & 0.97 & $(0.52516,0.69857)$ & 0.1734 & 0.99 \\
\hline \multirow{3}{*}{4} & & & $46, \quad i=1$ & $(0.92065,1.33125)$ & 0.4105 & 0.96 & $(0.95979,1.238)$ & 0.2782 & 0.99 \\
\hline & 80 & $A$ & $0, \quad i=2,3$ & $(0.5603,0.80458)$ & 0.2442 & 0.95 & $(0.56491,0.77644)$ & 0.2115 & 0.99 \\
\hline & & & $34, \quad i=4$ & $(0.52242,0.71787)$ & 0.1954 & 0.95 & $(0.53619,0.7074)$ & 0.1712 & 0.97 \\
\hline \multirow{3}{*}{4} & & & $60, \quad i=1$ & $(0.94692,1.27672)$ & 0.329 & 0.97 & $(1.03368,1.14761)$ & 0.1139 & 1 \\
\hline & 120 & $D$ & $0, \quad i=2,3$ & $(0.5783,0.77724)$ & 0.1989 & 0.95 & $(0.61165,0.71582)$ & 0.1041 & 1 \\
\hline & & & $60, \quad i=4$ & $(0.53343,0.70122)$ & 0.1678 & 0.97 & $(0.55972,0.65415)$ & 0.0944 & 1 \\
\hline \multirow{3}{*}{4} & & & $69, \quad i=1$ & $(0.9509,1.28246)$ & 0.3315 & 0.96 & $(1.03338,1.14746)$ & 0.1140 & 1 \\
\hline & 120 & $A$ & $0, \quad i=2,3$ & $(0.58608,0.78656)$ & 0.2004 & 0.91 & $(0.61344,0.71919)$ & 0.1057 & 1 \\
\hline & & & $51, \quad i=4$ & $(0.54107,0.70237)$ & 0.1613 & 0.92 & $(0.56179,0.65561)$ & 0.0938 & 0.99 \\
\hline
\end{tabular}

Table 6.6. NACIs and BCIs of $\left(\alpha, b, \theta_{1}\right)$ with their lengths (LNACIs and LBCIs) and coverage probabilities (CPNACIs and $C P B C I s)$. The population parameters values are $\left(\alpha=1.1, b=0.66\right.$ and $\left.\theta_{1}=0.6\right)$, values of the prior parameters $\left(\mu_{1}=12.1\right.$, $\mu_{2}=4.356, \mu_{3}=3.6 \lambda_{1}=11, \lambda_{2}=6.6$ and $\left.\lambda_{3}=6\right)$ and $\psi_{k}=0.01$.

\begin{tabular}{|c|c|c|c|c|c|c|c|c|c|}
\hline \multirow{4}{*}{$k$} & \multirow{4}{*}{$n$} & \multirow{4}{*}{ criterion } & \multirow{4}{*}{$n_{i}$} & \multicolumn{3}{|c|}{ NACIs } & \multicolumn{3}{|c|}{$B C I s$} \\
\hline & & & & $N A C I(\alpha)$ & $L N A C I(\alpha)$ & $C P N A C I(\alpha)$ & $B C I(\alpha)$ & $L B C I(\alpha)$ & $C P B C I(\alpha)$ \\
\hline & & & & $N A C I(b)$ & $L N A C I(b)$ & $C P N A C I(b)$ & $B C I(b)$ & $L B C I(b)$ & $C P B C I(b)$ \\
\hline & & & & $N A C I\left(\theta_{1}\right)$ & $L N A C I\left(\theta_{1}\right)$ & $C P N B C I\left(\theta_{1}\right)$ & $B C I\left(\theta_{1}\right)$ & $L B C I\left(\theta_{1}\right)$ & $C P B C I\left(\theta_{1}\right)$ \\
\hline \multirow{3}{*}{4} & & & $20, \quad i=1$ & $(0.85797,1.46016)$ & 0.6021 & 0.96 & $(0.85942,1.39068)$ & 0.5312 & 0.98 \\
\hline & 40 & $D$ & $0, \quad i=2,3$ & $(0.57752,0.7451)$ & 0.1675 & 0.94 & $(0.56739,0.74691)$ & 0.1795 & 0.95 \\
\hline & & & $20, \quad i=4$ & $(0.44655,0.74709)$ & 0.3005 & 0.93 & $(0.46458,0.77891)$ & 0.3143 & 0.97 \\
\hline \multirow{3}{*}{4} & & & $28, \quad i=1$ & $(0.85775,1.46274)$ & 0.6049 & 0.95 & $(0.85967,1.39187)$ & 0.5322 & 0.97 \\
\hline & 40 & $A$ & $0, \quad i=2,3$ & $(0.57579,0.75838)$ & 0.1825 & 0.92 & $(0.55226,0.75275)$ & 0.2004 & 0.95 \\
\hline & & & $12, \quad i=4$ & $(0.46773,0.73106)$ & 0.2633 & 0.91 & $(0.47936,0.75378)$ & 0.2744 & 0.97 \\
\hline \multirow{3}{*}{4} & & & $40, \quad i=1$ & $(0.9252,1.33879)$ & 0.4135 & 0.95 & $(0.96285,1.24218)$ & 0.2793 & 1 \\
\hline & 80 & $D$ & $0, \quad i=2,3$ & (0.60191, 0.72203) & 0.1201 & 0.94 & $(0.60209,0.71678)$ & 0.1146 & 0.96 \\
\hline & & & $40, \quad i=4$ & $(0.49335,0.71142)$ & 0.2180 & 0.95 & $(0.51436,0.70391)$ & 0.1895 & 0.98 \\
\hline \multirow{3}{*}{4} & & & $57, \quad i=1$ & $(0.92733,1.34129)$ & 0.4139 & 0.94 & $(0.9636,1.24367)$ & 0.2800 & 0.99 \\
\hline & 80 & $A$ & $0, \quad i=2,3$ & $(0.59739,0.7299)$ & 0.1325 & 0.94 & $(0.59258,0.72235)$ & 0.1297 & 0.96 \\
\hline & & & $23, \quad i=4$ & $(0.50562,0.69403)$ & 0.1884 & 0.95 & $(0.52125,0.69017)$ & 0.16889 & 0.97 \\
\hline \multirow{3}{*}{4} & & & $60, \quad i=1$ & $(0.95505,1.28823)$ & 0.3331 & 0.95 & $(1.03498,1.14947)$ & 0.1144 & 1 \\
\hline & 120 & $D$ & $0, \quad i=2,3$ & $(0.61526,0.71398)$ & 0.0987 & 0.96 & $(0.6248,0.69688)$ & 0.0720 & 0.99 \\
\hline & & & $60, \quad i=4$ & $(0.51907,0.70078)$ & 0.1817 & 0.95 & $(0.55475,0.65309)$ & 0.0983 & 1 \\
\hline \multirow{3}{*}{4} & & & $85, \quad i=1$ & $(0.9508,1.28275)$ & 0.3319 & 0.95 & $(0.97482,1.22413)$ & 0.2493 & 0.99 \\
\hline & 120 & $A$ & $0, \quad i=2,3$ & $(0.60642,0.71546)$ & 0.1090 & 0.97 & $(0.60327,0.71052)$ & 0.1072 & 0.97 \\
\hline & & & $35, \quad i=4$ & $(0.52081,0.67668)$ & 0.1558 & 0.94 & $(0.53165,0.67517)$ & 0.1435 & 0.97 \\
\hline
\end{tabular}

From Tables 6.1 6.6, the following observations can be made.

1. The MSEs of MEs, LSEs, MLEs and BEs of the considered parameters decrease as the sample size increases.

2. The BEs of $\alpha, b$ and $\theta_{1}$ give more accurate results through the MSEs than MLEs, MEs and LSEs.

3. The MLEs of $\alpha, b$ and $\theta_{1}$ give more accurate results through the MSEs than MEs and LSEs.

4. The LSEs of $b$ give more accurate results through the MSEs than MEs while MEs of $\alpha$ give more accurate results through the MSEs than LSEs.

5. The LNACIs and LBCIs decrease as the sample size increases.

6. The BCIs of $\alpha, b$ and $\theta_{1}$ give more accurate results than NACIs through the lengths of CIs.

7. The CPBCIs and CPNACIs are close to the nominal value (95\%).

8. The BCIs of $\alpha, b$ and $\theta_{1}$ have large coverage probabilities compared with NACIs. 

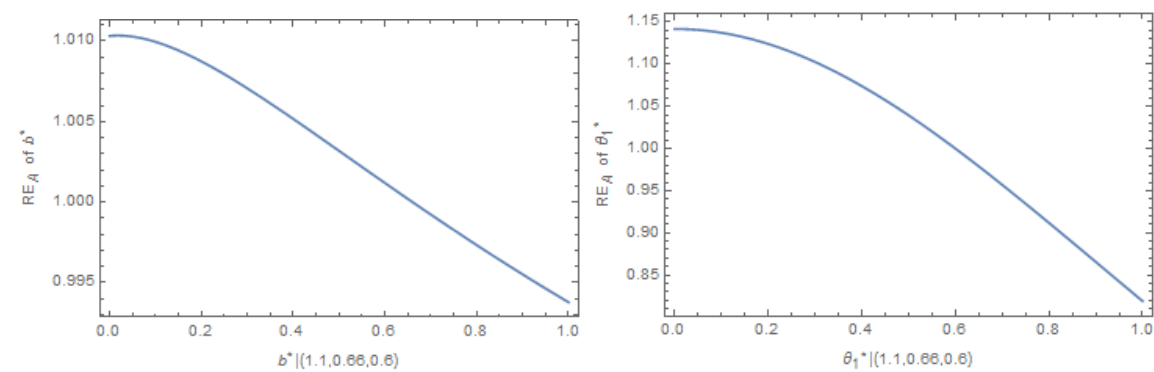

Figure 3: Relative efficiency against varying values of the parameters under A-optimality for the true values $\left(\alpha^{0}, b^{0}, \theta_{1}^{0}\right)=$ $(1.1,0.66,0.6)$.
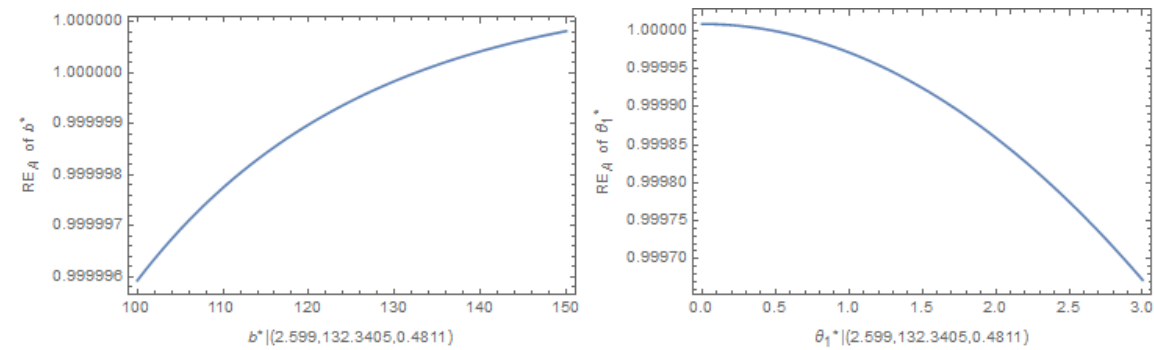

Figure 4: Relative efficiency against varying values of the parameters under A-optimality for the true values $\left(\alpha^{0}, b^{0}, \theta_{1}^{0}\right)=$ $(2.6268,134.053,0.4699)$.

\section{Sensitivity analysis}

The sensitivity analysis identifies the sensitive parameters which need to be estimated with special care for the purpose of minimizing the risk of obtaining an erroneous optimal solution. Our D-optimal plan is independent of the parameters $\alpha, b$ and $\theta_{1}$. That is, the D-optimal plan is very robust. On the other hand, our A-optimal plan depends on the two parameters $b$ and $\theta_{1}$. As a result, in this section, we consider a sensitivity analysis of the A-optimal plan for the sensitive parameters $b$ and $\theta_{1}$.

Let $\alpha^{0}, b^{0}$ and $\theta_{1}^{0}$ are the true values of the parameters $\alpha, b$ and $\theta_{1}$, respectively. Further, let $\alpha^{*}, b^{*}$ and $\theta_{1}^{*}$ be the specified values of the unknown parameters. Then, we can define the A-optimal relative efficiency of the specified values relative to the true values as follows:

$$
R E_{A}\left(b^{*}\right)=\frac{\operatorname{tr}\left(\mathbf{F}^{-1}\left(\alpha^{0}, b^{0}, \theta_{1}^{0}\right)\right)}{\operatorname{tr}\left(\mathbf{F}^{-1}\left(\alpha^{0}, b^{*}, \theta_{1}^{0}\right)\right)},
$$

and

$$
R E_{A}\left(\theta_{1}^{*}\right)=\frac{\operatorname{tr}\left(\mathbf{F}^{-1}\left(\alpha^{0}, b^{0}, \theta_{1}^{0}\right)\right)}{\operatorname{tr}\left(\mathbf{F}^{-1}\left(\alpha^{0}, b^{0}, \theta_{1}^{*}\right)\right)} .
$$

To examine the sensitivity of the A-optimal allocations in term of relative efficiency, we plot the A-optimal relative efficiency for two settings of the true parameters. The first setting is $\left(\alpha^{0}, b^{0}, \theta_{1}^{0}\right)=(1.1,0.66,0.6)$ with $\psi_{k}=0.1$ which used in the simulation study. The second setting is $\left(\alpha^{0}, b^{0}, \theta_{1}^{0}\right)=(2.6268,134.053,0.4699)$ with $\psi_{k}=0.99$ which used in the real data example. We compute the relative efficiencies of one parameter while keeping the others fixed. Plots of the relative efficiencies against the changing values of that parameters are presented in Figures 3 and 4

We note that when the values of the three parameters $\alpha, b$, and $\theta_{1}$ are not far removed from the true values, the changing in the relative efficiencies is not significantly large. Thus, the optimal allocation schemes are not sensitive to misspecification of the model parameters as long as they are not far deviated from the true values. 


\section{Conclusion}

This article has investigated some inferences on multiple ramp-stress ALT when the lifetime of a product follows the GHN distribution. We have obtained the MEs, LSEs, MLEs and BEs of the considered parameters and studied their performance via simulated. Normal approximation CIs and credible intervals were constructed for the considered parameters. From the results we recommend the use of maximum likelihood and Bayes methods in estimating the parameters under the consideration rather than moment and least square methods. We recommend the D- and A-optimality criteria to determine the optimal proportion of test units allocated to each stress level for the GHN distribution.

\section{References}

[1] J. I. McCool, (1980). Confidence limits for Weibull regression with censored data, IEEE Trans. Reliab., 29, 145-150.

[2] R. Miller, and W. Nelson, (1983). Optimum simple step-stress plans for accelerated life testing, IEEE Trans. Reliab., 32, 59-65.

[3] W. Nelson, (1990). Accelerated Testing: Statistical Models, Test Plans and Data Analysis, Wiley, New York.

[4] A. H. Abdel-Hamid, (2009). Constant-partially accelerated life tests for Burr type-XII distribution with progressive type-II censoring, Comput. Stat. Data Anal., 53, 2511-2523.

[5] Z. F. Jaheen, H. M. Moustafa, and G. H. Abd El-Monem, (2014). Bayes inference in constant partially accelerated life tests for the generalized exponential distribution with progressive censoring, Commun. Stat. Theor. M., 43, 2973-2988.

[6] M. M. Mohie El-Din, S. E. Abu-Youssef, N. S. A. Ali, and A. M. Abd El-Raheem, (2016). Estimation in constant-stress accelerated life tests for extension of the exponential distribution under progressive censoring, Metron, 74(2), 253-273.

[7] M. M. Mohie El-Din, S. E. Abu-Youssef, N. S. A. Ali, A. M. Abd El-Raheem, (2018). Inference on constant-stress accelerated life testing based on geometric process for extension of the exponential distribution under type-II progressive censoring, Pak. J. Stat. Oper. Res., 14(2), 233-251.

[8] N. Balakrishnan, and D. Han, (2008). Exact inference for a simple step stress model with competing risks for failure from exponential distribution under type-II censoring, J. Stat. Plan. Inf., 138, 4172-4186.

[9] M. M. Mohie El-Din, S. E. Abu-Youssef, N. S. A. Ali, and A. M. Abd El-Raheem, (2015). Estimation in step-stress accelerated life tests for Weibull distribution with progressive first-failure censoring, J. Stat. Appl. Pro., 3, 403-411.

[10] M. M. Mohie El-Din, S. E. Abu-Youssef, N. S. A. Ali, and A. M. Abd El-Raheem, (2015). Estimation in step-stress accelerated life tests for power generalized Weibull distribution with progressive censoring, Adv. Stat., 2015, 1-13.

[11] M. M. Mohie El-Din, S. E. Abu-Youssef, N. S. A. Ali, and A. M. Abd El-Raheem, (2016). Parametric inference on stepstress accelerated life testing for the extension of exponential distribution under progressive type-II censoring, Commun. Stat. Appl. Methods, 23(4), 269-285.

[12] A. H. Abdel-Hamid, and E. K. AL-Hussaini, (2007). Progressive stress accelerated life tests under finite mixture models, Metrika, 66, 213-231.

[13] A. H. Abdel-Hamid, and E. K. AL-Hussaini, (2011). Inference for a progressive stress model from Weibull distribution under progressive type-II censoring, J. Comput. Appl. Math., 235, 5259-5271.

[14] E. K. AL-Hussaini, A. H. Abdel-Hamid, and A. F. Hashem, (2015). One-sample Bayesian prediction intervals based on progressively type-II censored data from the half-logistic distribution under progressive stress model, Metrika, 78, 771-783.

[15] A. H. Abdel-Hamid, and T. A. Abushal, (2015). Inference on progressive-stress model for the exponentiated exponential distribution under type-II progressive hybrid censoring, J. Stat. Comput. Simul., 85, 1165-1186.

[16] M. M. Mohie El-Din, S. E. Abu-Youssef, N. S. A. Ali, and A. M. Abd El-Raheem, (2017). Classical and Bayesian inference on progressive-stress accelerated life testing for the extension of the exponential distribution under progressive type-II censoring, Qual. Reliab. Engng. Int., 33, 2483-2496. 
[17] D. S. Bai, M. S. Kim, and S. H. Lee, (1989). Optimum simple step-stress accelerated life tests with censoring, IEEE Trans. Reliab., 38, 528-532.

[18] I. H. Khamis, and J. J. Higgins, (1996). Optimum 3-step-stress tests, IEEE Trans. Reliab., 45, 341-345.

[19] I. H. Khamis, (1997). Comparison between constant and step-stress tests for Weibull models, Int. J. Qual. Reliab. Manag., $14,74-81$.

[20] I. H. Khamis, and J. J. Higgins, (1998). A new model for step-stress testing, IEEE Trans. Reliab., 47, 131-134.

[21] H. K. T. Ng, N. Balakrishnan, and P. S. Chan, (2007). Optimal sample size allocation for tests with multiple levels of stress with extreme value regression, Nav. Res. Log., 54, 237-249.

[22] P. W. Srivastava, and R. Shukla, (2008). A log-logistic step-stress model, IEEE Trans. Reliab., 57, 431-434.

[23] P. W. Srivastava, and R. Shukla, (2008). Optimum log-logistic step-stress model with censoring, Int. J. Qual. Reliab. Manag., 25, 968-976.

[24] P. W. Srivastava, and N. Mittal, (2010). Optimum step-stress partially accelerated life tests for truncated logistic distribution with censoring, Appl. Math. Modell., 34, 3166-3178.

[25] D. Han, and H. K. T. Ng, (2013). Comparison between constant-stress and step-stress accelerated life tests under time constraint, Nav. Res. Log., 60, 541-556.

[26] Q. Guan, Y. Tang, J. Fu, and A. Xu, (2014). Optimal multiple constant-stress accelerated life tests for generalized exponential distribution, Commun. Stat. Simul. Comput., 43, 1852-1865.

[27] D. Han, (2015). Time and cost constrained optimal designs of constant-stress and step-stress accelerated life tests, Reliab. Eng. Syst. Saf., 140, 1-14.

[28] M. M. Mohie El-Din, S. E. Abu-Youssef, N. S. A. Ali, and A. M. Abd El-Raheem, (2017). Optimal plans of constant-stress accelerated life tests for the Lindley distribution, J. Test. Eval., 45(4), 1463-1475.

[29] A. M. Abd El-Raheem, (2019). Optimal plans of constant-stress accelerated life tests for extension of the exponential distribution, J. Test. Eval., 47(2), (in press).

[30] A. M. Abd El-Raheem, (2019). Optimal plans and estimation of constant-stress accelerated life tests for the extension of the exponential distribution under type-I censoring, J. Test. Eval., 47(5), (in press).

[31] K. Cooray, and M. M. A. Ananda, (2008). A generalization of the half-normal distribution with applications to lifetime data, Commun. Stat. Theor. M., 37, 1323-1337.

[32] K. Ahmadi, M. Rezaei, and F. Yousefzadeh, (2013). Estimation for the generalized half-normal distribution based on progressive type-II censoring, J. Stat. Comput. Simul., 85, 1128-1150.

[33] N. M. Olmos, H. Varela, H. Bolfarine, et al., (2014). An extension of the generalized half-normal distribution, Stat. Pap., $55,967-981$.

[34] L. Wang, and Y. Shi, (2017). Estimation for constant-stress accelerated life test from generalized half-normal distribution, J. Syst. Eng. Electronics, 28, 810-816.

[35] D. S. Bai, M. S. Cha, and S. W. Chung, (1992). Optimum simple ramp-tests for the Weibull distribution and type-I censoring, IEEE Trans. Reliab., 41, 407-713.

[36] P. W. Srivastava, and D. Sharma, (2015). Optimum time-censored simple ramp-stress accelerated life test sampling plan for the log-logistic distribution, JQME, 21, 112-132.

[37] R. Miller, (1981). Survival Analysis, Wiley, New York.

[38] Y. Zhu, (2010). Optimal Design and Equivalency of Accelerated Life Testing Plans, PhD thesis, The State University of New Jersey, United States. 